
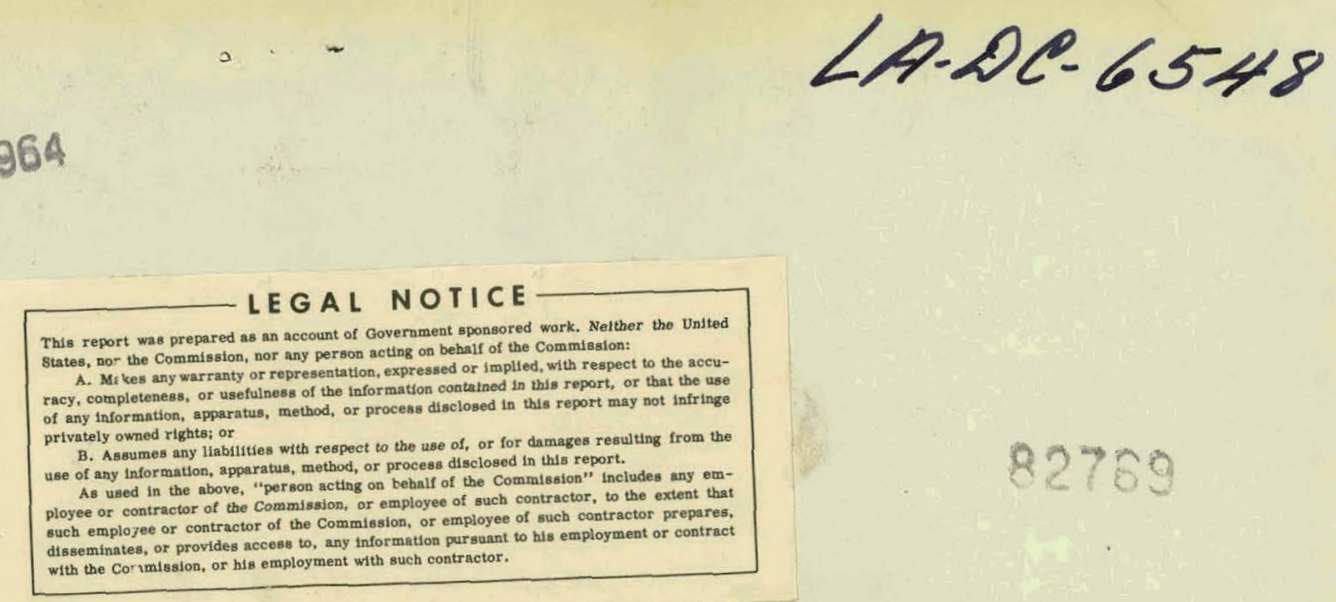

This paper was submitted for publication in the open literature at least $\sigma^{\prime}$ months prior to the issuance date of this Microcard. Since the U.S.A.E.C. has no evidence that it has been published, the paper is being distributed in Microcard form as a preprint.

\section{COUPLING CONSIDERATIONS IN TWO-ELECTRON SPECTRA*}

by

Robert D. Cowan University of California

Los Alamos Scientific Laboratory Los Alamos, New Mexico and

Kenneth L. Andrew Department of Physics Purdue University Lafayette, Indiana

\title{
ABSTRACT
}

The energy level structure, relative line strengths, and Landé g-factors of two-electron configurations are discussed for four important types of pure coupling LS, LK, $j K(j l)$, and $j j$. Transitions from one type of coupling to another are discussed in detail, using the configuration pf as an example. The appropriateness of IS- and jj-coupling notation in two-electron spectra is quite limited for atoms of medium atomic weight, where nearly all excited configurations show a strong tendency toward pair ( $\mathrm{LK}$ to $j K$ ) coupling. For other atoms, pair coupling occurs mainly for high values of orbital angular momentum of the excited electron; the coupling may be near LK for small 


\section{DISCLAIMER}

This report was prepared as an account of work sponsored by an agency of the United States Government. Neither the United States Government nor any agency Thereof, nor any of their employees, makes any warranty, express or implied, or assumes any legal liability or responsibility for the accuracy, completeness, or usefulness of any information, apparatus, product, or process disclosed, or represents that its use would not infringe privately owned rights. Reference herein to any specific commercial product, process, or service by trade name, trademark, manufacturer, or otherwise does not necessarily constitute or imply its endorsement, recommendation, or favoring by the United States Government or any agency thereof. The views and opinions of authors expressed herein do not necessarily state or reflect those of the United States Government or any agency thereof. 


\section{DISCLAIMER}

Portions of this document may be illegible in electronic image products. Images are produced from the best available original document. 
values of the principle quantum number of this electron, and it approaches : pure $j K$ as this quantum number increases. It is emphasized that LK or $j K$ notation would best be used only when the coupling conditions approximate LK or $j K$ coupling, respectively; either notation can serve to unambigously Identify levels throughout the range of intermediate pair couplings, but will not correctly designate the nature of the quantum states because of reversals of the $L$ and $f$ compositions of certain states as the coupling conditions change from pure LK to pure JK. 


\section{I: Introduction}

For many years the analysis and interpretation of atomic spectra were based almost entirely on the concepts of LS and $j J$ coupling. More recently there has been increasing recognition of the importance of other types of coupling, particularly in the more highly excited configurations. Racah, especially, bas emphasized the desirability of denoting observed energy levels in terms of the pure-coupling scheme which most nearly approximates the coupling conditions actually present. ${ }^{1}$ In splte of this, there seems to have been a great deal of reluctance to give up IS notation even when clearly inappropriate. There also has developed some confusion In terminology in the literature, and the unwary user may easily misinterpret what he reads. Both of these are probably due partly to relative unfamiliarity with the exact nature of the newer coupling schemes and their relation to $I S$ and $j j$ coupling. One of the main purposes of this paper is to try to rectify this situation by undertaking a thorough discussion of coupling considerations in two-electron configurations, with emphasis on pair coupling. The theoretical backeround material required Is largely old and well-known to workers in theoretical atomic structure, but it is widely scattered in the literature and difficult for the unin- 
Itiated to dig out. Accordingly, we have provided a fairly complete bibliography and discussed the older couplings as well as the new ones for comparative purposes and orientation of the experimental spectroscopist. 


\section{Qualitative Considerations}

The simplest possible complex atomic spectra are those arising from electronic configurations consisting of only two electrons outside of closed shells or subshells. Although comparatively simple, the number of atoms exhibiting basically two-electron spectra is by no means trivial, since these include not only He and the alkaline earth elements (ground configuration $\mathrm{ns}^{2}$ ); but also the fourth-period elements $C, \mathrm{~S} 1, \mathrm{Ge}, \mathrm{Sn}$, and $\mathrm{Pb}$ (ground configurations $\mathrm{ns}^{2} \mathrm{np}^{2}$, most excited configurations leaving the $n s^{2}$ subshell intact); the rare gases other than He (whose excited configurations $\mathrm{p}^{5} l$ are very similar to $\mathrm{pl}$ ); the third spectra of $\mathrm{Ti}, \mathrm{Zr}, \mathrm{Hf}$, and Th (ground configuration $\mathrm{d}^{2}$ ); spectra such as those of $\mathrm{Cu} I I, P d I$, $\mathrm{Ag} I I$, and $\mathrm{Au}$ II (whose excited configurations $d^{9} l$ are similar to $d l$ ); Ce III (ground configuration $f^{2}$ ); and of course ions isoelectronic to all of the above. In addition, any configuration of the type $l_{a}^{\alpha} l_{b}^{\beta} \cdots l_{n^{2}}^{\nu}$ (where the electron $l_{2}$ is weakly bound to the core $l_{a^{2}}^{\alpha} l_{b} \cdots l_{n}^{v}$ ) bears many similarities to the two-electron configuration $\ell_{2} l_{2}$, the resultant values $L_{1}$ and $S_{1}$ for the core replacing $l_{1}$ and $s_{1}$; much of the discussion of this paper is applicable by simple generalization to all such configurations. 
For the ground and low excited configurations it is well known tinat the coupling of the four angular momentum vectors $l_{1} s_{1} l_{2} s_{2}$ is usually best described for elements of $10 \mathrm{w}$ and medium atomic number by LS coupling

$$
\left[\left(\ell_{1} \ell_{2}\right) L,\left(s_{1} B_{2}\right) S\right] J,
$$

and for elements of high atomic number by $j j$ coupling

$$
\left[\left(L_{1} s_{1}\right) j_{1},\left(l_{2} s_{2}\right) j_{2}\right] J
$$

that is to say, for the low- and medium- $Z$ elements the electrostatic interaction between electrons is predominant, whereas for high- 2 elements it is the magnetic interaction between the spin of each electron and its own orbital motion which predominates.

When the outer electron is more highly excited, and particularly when it has a high value of $l$, the coupling is usually no longer close to efther IS or jJ. One should not be surprised at this; after all, the couplings (1) and (2) are symmetric in the two electrons whereas the configurations in question are obviously highly asymetric. Along with the departures from LS and $j j$ coupling conditions, there are pronounced changes in the level structures. Observed structures frequently show a striking tendency of the levels to occur in pairs, with the J-values of the levels of each pair differing by unity; an examle was early pointed out by Shenstone $e^{2}$ in connection with the analysis of the $d^{8} g$, and to a 
lesser extent $d^{\theta} f$, conflgurations of $\mathrm{Cu}$ II. This pairing, together with that of simflar cases in the rare-gas configurations $p^{5} d$ and $p^{5} f$, was explained theoretically by Shortley and Fried, ${ }^{3}$ using the Slater tineory of atomic structure 4 with simplifications now known as the Shortley-Fried approximation. Close pairing of energy levels has more recently been observed in configurations such as $\mathrm{pf}$ and $\mathrm{pg}$ in $\mathrm{N} \mathrm{II,},{ }^{6} \mathrm{Si} I,^{7} \mathrm{~S} 1$ III, ${ }^{8}$ $P$ II $,{ }^{\theta} \mathrm{Ge} \mathrm{I}, 10 \mathrm{As}$ II, ${ }^{11}$ and Sn I, ${ }^{12} \mathrm{dg}$ and $\mathrm{dh}$ in Ti III, ${ }^{13} \mathrm{fg}$ in Ce III, ${ }^{14}$ $p^{2} f$ in $N^{15}$ and $O I I,{ }^{18}$ and $p^{4} f_{\text {and }} p^{4} g$ in $A I^{17}$ and $C l I^{28}$; in several cases the level structure has been discussed in terms of the "pair-coupline approximation," which is a less extreme approximation tinan the ShortleyFried treatment.

Racah ${ }^{19}$ pointed out many years ago that the conditions responsible for this pairing were frequently such as to approximate a third type of vector coupling

$$
\left[\left(\left(l_{1} s_{2}\right) J_{1}, l_{2}\right) K, s_{2}\right] J
$$

That is, when the outer electron is sufficiently highly excited its interaction with the inner electron becomes so small that the spin-orbit. interaction $\left(l_{1} s_{2}\right)$ of the inner electron predominates; at the same time, the spin-orbit interaction of the outer electron $\left(l_{2} s_{2}\right)$ is so weak (especially for large $l$, going roughly as $\left.\left(n_{2} l_{2}\right)^{-3}\right)^{20}$ that the $\left(l_{1} l_{2}\right)$ interaction is the second-most important one. This condition we shall refer to as $J K$ coupling by analogy with $L S$ and $j J$ coupling - $j$ and $K$ 
being the two Intermediate quantum numbers whose values, together with those of $J$ and $M$, completely specify the (pure-coupling) state of any given two-electron configuration. Though Racah introduced this coupling over twenty years ago (under the name $j l$ coupling, which is the terminology usually used in the literature), it has become widely known and used only within the last decade.

Outside the spectroscopy group at Lund, it still sppears to be practically unrecognized that in the light elements the spin-orbit interaction $\left(\ell_{2} s_{2}\right)$ may be so small that for not-too-highly exclted states the interaction $\left(l_{1} l_{2}\right)$ may be the larger of the two, the $\left(l_{2} s_{2}\right)$ and $\left(s_{1} s_{2}\right)$ interactions still being small. We have then a fourth important type of coupling,

$$
\left[\left(\left(l_{1} l_{2}\right) L, s_{i}\right) K, s_{2}\right] J
$$

LK-coupling notation has been used very little in the literature, $6,8,15,18$ and then sometimes when JK notation would be rather more appropriate (see Sec. VI).

Because of the weak $s_{2}$ interactions there is a pronounced pairing of energy levels under LK-coupling conditions, similar to that of the $j K$ case. Indeed, both (3) and (4) are just special cases of the more general coupling

$$
\left[\left(\ell_{1} l_{2} s_{1}\right) K, s_{2}\right] J
$$


In which the coupling of $s_{2}$ to $K$ is weak 60 that $K$ is a good quantum number, but in which the relative importance of the interactions $\left(l_{1} l_{2} s_{1}\right)$ 1s arbitrary. Such a situation exists whenever the $\left(\ell_{2} s_{2}\right)$ and $\left(s_{2} s_{2}\right)$ Interactions are small compared with $\left(l_{1} l_{2}\right)$ and $\left(l_{1} s_{1}\right)$. Throughout the range of "Intermediate pair coupling" conditions"15,18 in which neither $\left(l_{1} l_{2}\right)$ nor $\left(l_{1} s_{1}\right)$ is large compared wh the other, we still have a paired level structure. This arises solely because of the ascumed weak coupling of $\mathrm{s}_{2}$ to $\mathrm{K}$, and is simply a generalization of the situation existing in any single-electron doublet spectrum (where the analogs of the quantum numbers $K, s_{2}, J$ are $\left.l, s, J\right)$. .

Because the concept of $L K$ coupling is so new, the term "pair coupling" has frequently been used synonymously with "jk coupling," $1,17,21,22$ even in the quite recent literature. This is clearly an unnecessarily restricted use of "palr coupling," which should in the future be considered synonymous wh the more general coupling (5) and with "the paircoupling approximation" $\left(\left(l_{2} s_{2}\right)\right.$ and $\left.s_{1} s_{2}\right)$ negligible). It is really "the Shortley-Frled approximation" $\left(\left(\ell_{2} s_{2}\right)\right.$ and $\left.s_{1} s_{2}\right)$ negligible, plus $\left(\ell_{1} \ell_{2}\right)$ small compared with $\left.\left(l_{1} s_{1}\right)\right)$ which corresponds specifically to $j K$ coupling.

It is convenient to discuss the detailed relationships among the four above types of coupling by means of specific numerical examples. To do this it is necessary to obtain expressions for the transformation and energy matriceg involved. 


\section{Basis Transformations}

We Pirst introduce four sets of basis wavefunctions

$$
\text { (LSJM), (LKNM), } \left.\left.\quad \mid j_{1} K J M\right), \quad \text { and } \mid j_{2} j_{2} \pi M\right) \text {, }
$$

each set corresponding to one of the pure-coupling cases (1)-(4), and each wavefunction being an eigenfunction of the four angular-momentum operators indicated. The actual wavefunction for any state (BJM) can then be expanded in terms of one of these four sets; for example,

$$
(\beta J M)=\sum_{L S}(L S J M)(L S J \mid \dot{B J}) \equiv \sum_{L S}(L S J M) B_{L S J}^{3},
$$

where in the absence of external fields $J$ is a rigorous quantum number, and where orthonormalization of the $(\beta J \mu)$ requires that the expansion coefficents (LSJ|BJ) be elements of a unitary (usually, real orthogonal) matrlx $U$. We have assumed here the common approximation in which the .. basis sets (6) include only the states of a single configuration $l_{1} l_{2}$ of 1nterest, and will mostly drop the $M$ quantum number. 23 
In particular, (7) can represent the expansion of one set of basis functions in terms of another, and the corresponding transformation matrices are of special interest. The simplest to calculate are those between the $j K$ and $j J$ representations and between the LK and LS representations, because each corresponds to a simple recoupling of three angular momenta; the matrix elements have been calculated by Racah, ${ }^{24}$ and in terms of 6-J symbols $8^{25-27}$ are:

$$
\begin{aligned}
& \mathrm{U}_{j_{1} K, j_{2} j_{2}} \equiv\left(\left(j_{1} \ell_{2}\right) K, s_{2} J \mid j_{1},\left(\ell_{2} s_{2}\right) j_{2}, J\right) \\
& =(-1)^{j_{1}+l_{2}+\sigma_{2}+J}[K]^{\frac{1}{2}}\left[j_{2}\right]^{\frac{1}{2}}\left\{\begin{array}{lll}
K & s_{2} & J \\
j_{2} & j_{1} & l_{2}
\end{array}\right\}, \\
& U_{L K, L S} \equiv\left(\left(L_{1}\right) K, s_{2} J \mid L,\left(s_{1} s_{2}\right) S, J\right) \\
& =(-1)^{I+s_{2}+s_{2}+J}[K]^{\frac{1}{3}}[E]^{\frac{1}{2}}\left\{\begin{array}{lll}
K & s_{2} & J \\
S & L & s_{2}
\end{array}\right\},
\end{aligned}
$$

where

$$
[K] \equiv 2 K+1 \text {, etc. }
$$

Using the Clebsch-Gordon-coefficlent phase relation ${ }^{28}$ 


$$
\left.\left.\mid\left(g_{2} g_{2}\right) g_{3}\right)=(-1)^{j_{1}+j_{2}-j_{3}} \mid\left(g_{2} j_{1}\right) j_{3}\right)
$$

we obtain similarly

$$
\begin{aligned}
U_{L K, J_{1} K} & =(-1)^{-l_{2}-l_{2}+L+j_{1}+l_{2}-K}\left(\left(l_{2} l_{1}\right) L, s_{1} K / l_{2},\left(l_{2} s_{2}\right) j_{1}, K\right) \\
& \left.=(-1)^{L+J_{1}+l_{2}+s_{1}}[L]^{\frac{1}{2}}\left[J_{1}\right]^{\frac{2}{2}} \mid \begin{array}{lll}
L & s_{1} K \\
j_{2} & l_{2} & l_{2}
\end{array}\right) .
\end{aligned}
$$

The remaining transformation matrices are given by appropriate matrix products of the above expressions. $U_{L S, J_{1} K}$ and $U_{L K, j_{2} j_{2}}$ prove to be simple products of (12) with (9) and (8), respectively. The LS-jj transformation matrix may be written

$$
\begin{aligned}
& U_{L S, j_{2} J_{2}}=\sum_{K} U_{L S, J_{2}} K^{U} J_{2} K, J_{2} J_{2} \\
& =\left([L][S]\left[J_{1}\right]\left[J_{2}\right]\right)^{\frac{1}{2}} \sum_{K}(-1)^{2 K}[K]\left\{\begin{array}{lll}
K & s_{2} & J \\
J_{2} & J_{1} & l_{2}
\end{array}\right)\left(\begin{array}{lll}
K & s_{2} & J \\
S & L & s_{1}
\end{array}\right)\left(\begin{array}{lll}
L & s_{1} & K \\
J_{2} & l_{2} & l_{2}
\end{array}\right\} \\
& =\left([L][S]\left[J_{1}\right]\left[J_{2}\right]\right)^{\frac{1}{2}}\left\{\begin{array}{lll}
l_{1} & l_{2} & L \\
s_{1} & s_{2} & S \\
J_{1} & J_{2} & J
\end{array}\right\} \text {, }
\end{aligned}
$$


where use has been made of the fact that $2\left(J+s_{2}+K\right)$ is an even number; th1s is the usual expression for the transformation between the IS and ig representations in terms of a 9-j symbol. ${ }^{25-27}$ (Note: In the case of equivalent electrons, half of the otherwise-possible states with $j_{2} \neq j_{2}$ are forbldden by the Pauld principle, as are odd-J states with $j_{1}=j_{2}$ and all states for which $L+S$ is odd; all remalning matrix elements (13) for $j_{1} \neq j_{2}$ must be multiplied by an additional factor $\sqrt{2}$ to precerve normalization. $L K$ and $j K$ coupling are physically meaningless since tiney treat the two electrons differently, and so all other transformation matrices are to be ignored.) 


\section{Calculation of Energy Matrices}

The problem of calculating the level structure of the configuration 1s that of finding the elgenvalues of the electron-interaction Hamiltonian ${ }^{4}$

$$
H=H_{e l}+H_{m a g}=\sum_{j>i} \frac{e^{2}}{r_{i j}}+\sum_{i} \xi_{i}\left(r_{i}\right) \times\left(\vec{l}_{1} \cdot \vec{s}_{1}\right),
$$

where the sums are carried out over all electrons outside of closed (sub-) shells, and smaller terms concerned with orbit-orbit and spin-otiner-orbit magnetic interactions have been omitted. In the usual matrix formulation, one finds the eigenvalues of the matrix corresponding to (14) in some specific representation (one such matrix for each value of $J$, the matrix being valid for each of the $2 J+1$ values of $M$ ), the components of the eigenvectors being the expansion coefficients $B_{L S J}$, in the example (7). The electrostatic portion of (14) is most easily evaluated in the LS representation, where for a two-electron conflguration the matrix is completely dagonal with aiagonal elements

$$
\left(\mathrm{LS}\left|\mathrm{H}_{\mathrm{el}}\right| \mathrm{LS}\right)=\sum_{\mathrm{k}} \mathrm{f}^{\mathrm{k} \mathrm{F}^{\mathrm{k}}}+\mathrm{g}^{\mathrm{k} \mathrm{G}^{\mathrm{k}}}
$$


Here the $F^{k}$ and $G^{k}$ are certain radial integrals $s^{4,5}$ related respectively to the magnitudes of the $\left(l_{2} l_{2}\right)$ and $\left(s_{2} s_{2}\right)$ aspects of the electrostatic Interaction; they are commonly treated as empirically adjustable parameters. The coefficients of these parameters may be written ${ }^{21}$

$$
\begin{aligned}
& f^{k}=(-1)^{L}\left(2 l_{1}+1\right)\left(2 l_{2}+1\right)\left(\begin{array}{lll}
l_{1} & l_{1} & k \\
0 & 0 & 0
\end{array}\right)\left(\begin{array}{lll}
l_{2} & l_{2} & k \\
0 & 0 & 0
\end{array}\right)\left(\begin{array}{lll}
l_{1} & l_{2} & L \\
l_{2} & l_{2} & k
\end{array}\right\}, \\
& 8^{k}=(-1)^{S}\left(2 l_{1}+1\right)\left(2 l_{2}+1\right)\left(\begin{array}{lll}
l_{1} & l_{2} & k \\
0 & 0 & 0
\end{array}\right)^{2}\left\{\begin{array}{lll}
l_{1} & l_{2} & L \\
l_{1} & l_{2} & k
\end{array}\right),
\end{aligned}
$$

where the six-component quantities in parentheses are $3-j$ symbols. $25-27$ Note that though the $G^{k}$ arise from electrostatic interactions, the energy dependence on the $G^{k}$ is different for different total spin; this is the result of electron-correlation effects arising from the Pauli exclusion principle.

The magnetic portion of (14) is easily evaluated in the jj representation, where (for two-electron configurations) the matrix is diagonal with diagonal elements

$$
\left(j_{2} j_{2}\left|H_{\operatorname{mag}}\right| j_{1} j_{2}\right)=\sum_{1} d_{i} \delta_{i} .
$$

The $\zeta_{1}$ are radial integrals ${ }^{4}$ measuring the strength of the spin-orbit 
interactions $\left(L_{1} s_{1}\right)$, and are customarily treated as additional empirical parameters; the values of the coefficients are

$$
a_{1}= \begin{cases}\frac{1}{2} l_{1}, & j_{i}=l_{1}+\frac{1}{2}, \\ -\frac{1}{2}\left(l_{1}+1\right), & j_{i}=l_{1}-\frac{1}{2} .\end{cases}
$$

In the case of conflgurations $\ell_{2}^{\epsilon} \ell_{2}$ where $\ell_{1}^{\epsilon}$ is a complete subshell minus one electron, the coefficients $f^{k}$ and $d_{2}$ are the negatives of those for $\ell_{2} \ell_{2}, 4$ and the coefficients $g^{k}$ are 29,27 :

$$
\begin{aligned}
& \mathrm{g}^{\mathrm{k}}=0, \\
& g^{L}=\frac{2\left(2 l_{1}+1\right)\left(2 l_{2}+1\right)}{2 L+1}\left(\begin{array}{lll}
l_{1} & l_{2} & L \\
0 & 0 & 0
\end{array}\right)^{2}, \quad S=0 .
\end{aligned}
$$

The fact that most of the $g^{k}$ are thus zero results in a strong tendency toward pair coupling in the spectra of the noble gases (configurations $\mathrm{p}^{5} l$ ) and of $\mathrm{Cu} I I$, etc. $\left(d^{9} l\right)$.

Using avallable tables of the $3-j$ and $6-j$ symbols 27 and of the $9-j$ symbols ${ }^{30}$ there is no problem in principle in evaluating by hand the coefficlent matrices $(16),(17),(19),(20)^{31}$ and the transformation matrices (8)-(13), and in using the latter to transform the former to any of the desired four representations. However, this procedure is tedious 
and subject to human error. To avold the se we have developed a Fortran code (henceforth called RCEI) to do the detalled calculations on IBM 7094 computers; this code evaluates from standard algebralc expressions ${ }^{27}$ the $3 n-j$ symbols required, printing out all matrices for hand use, and also writing them on magnetic tape for further computer work.

As examples for use in later sections of this paper, we list in Tables AI-AIII the transformation and Hamiltonian matrices for the configurations pf and $p^{5} f$. The constant term $F_{o}$, occurring with unit coefficlent in every diagonal element bas been omltted for brevity, and the coefficients $f^{k}$ and $B^{k}$ have been converted to coefficients of the parametere $F_{k}$ and $G_{k}$ by multiplication with the usual constant factors $D_{k^{*}} 4,28$ 


\section{v. Coupling Considerations in pp}

As a quantitative example to lllustrate the qualitative discussion of Sec. II, we consider in some detall the conflguration pf, for which we have the electrostatic parameters $F_{2}, G_{2}$, and $G_{4}$, and the opin-orbit parameters $\zeta_{p}$ and $\zeta_{f^{*}}$ Since the effects of $G_{4}$ are usually gmall compared with those of $G_{2}, G_{4}$ will for simplicity be neglected throughout the following discusstoin.

In F1g. 1 we 1llustrate the development of the level structure of pf In both Is and LK coupling, the energies shown having been calculated from the matrices of Table AII for the indicated values of the parameters. At the extreme left of the figure is shown the effect of $F_{2}(=1)$ in producing a separation into three quadruply-degenerate ${ }^{23}$ levels characterized by I quantum numbers D, F, and $G$. Where the value of the abscissa $\xi=\delta_{p} /\left(50 G_{2}+\delta_{p}\right)$ is zero (pure LS coupling), we see the effect of $G_{2}(=0.06)$ in splitting each of these L levels into a singlet and a (degenerate) triplet. Finally, for slightly larger values of $\xi$, non-zero $\because x^{n+3}+3$, Fing values of $f_{p}$ remove the remaining degeneracy of the triplets (cf. Eq. (1)). 32 Similarly, at the right-hand edge of the figure where $\xi$ is unity (appraximate IK coupling), we see the effect of $C_{p}(-3)$ in splitting each 
L-level 1nto two sub-levels with $K=L \pm \frac{1}{2}$, each sub-level being doubly degenerate. Finally, for $\xi<1$, non-zero values of $G_{2}$ remove the final degeneracy 32 to produce the paired level structure characteristic of IK-(or of any pair-) coupling. (cf. Eq. (4).)

The transition from LS to LK coupling and the correlation of J-levels In the two extremes is shown in the central portion of the figure $0<\xi<1$, wh1ch has been drawn for the case $50 G_{2}+\zeta_{p}=$ constant $=3$. The numbers 50 and 3 are arbitray, chosen simply to provide convenient vertical scales. Analogously to the method frequently used in drawing similar figures 4,20 the non-linear restriction $\left[\left(50 G_{2}\right)^{2}+\sigma_{p}^{2}\right]^{\frac{1}{2}}=$ constant could have been used, but we have here preferred the "linear" plot (cf. reference 22, pp. $113 \mathrm{ff}$ ) for two ressons:

(1) Levels which do not interact with any others ( $J=1$ and $J=5$ In the present case) are straight lines.

(2) More importently, if the energy dependence on $\zeta_{p}$ were neglected the triplets would remain degenerate for all $\xi$, and the singlets and triplets would appear as straight lines converging to zero singlet-triplet splitting at $\xi=1$. Simllarly, if the energy dependence on $G_{2}$ were neglected, the degenerate doublets characterlzed by quantum numbers LK at $\xi=1$ would be (approximately) stralght lines converging to zero K-splitting at $\xi=0$. The method of plotting used here thus shows much more clearly than would the non-11near method the disappearance of the $S$ (singlettriplet) oplitting and the appearance of the $K$ splitting as one passes from $\bar{\zeta}=0$ to $\xi=1$. 
In Fig. 2 we show similarly the development of the characteristic level structure under $j J$-coupling conditions, $\xi \equiv \xi_{f} /\left(5 F_{2}+\zeta_{f}\right) \geq 1$, and the development of the paired level structure of $\mathrm{JK}$ coupling, $\xi \cong 0$. Again, the method of plotting used shows fairly clearly the disappearance of the $j_{2}$ oplitting and the appearance of the $K$ splitting as one passes from $\mathrm{jJ}$ to $\mathrm{JK}$ coupling conditions.

A feature which appears clearly in Fig. 2 (not apparent in Fig. 1) is an obvious interaction between the two upper $J=4$ levels and also between the two lower $J=3$ levels. For each palr, the general trend of the levels 16 clearly such that they would cross if they were not prevented from doing so by the interaction between them; this behavior of energy levels is well known. However, it is perhaps not commonly realized that the limiting compositions of the corresponding quantum states are as though the levels did cross. Consider, for example, the elgenvectors of the two highest $J=4$ levels in the $J f$ representation: In the limit $\xi=1$, these vectors are pure $\left(\frac{3}{2} \frac{7}{2}\right)_{4}$ and $\left(\frac{3}{2} \frac{5}{2}\right)_{4}$ states; as $\xi$ decreases, the purities of the vectors decrease unt1l at $\xi \cong 0.4$ each vector has nearly equal components of the two basis states; as $\xi$ approaches 0 , the vectors appear to become Increasingly pure again, but (at first thought, somewhat surprisingly) the vector associated with the highest $\mathrm{J}=4$ level is now primarily $\left(\frac{3}{2} \frac{5}{2}\right)_{4}$ whereas it was initially $\left(\frac{3}{2} \frac{7}{2}\right)_{4}$. Similarly, if we use the $j K$ representation, we find that the elgenvector belonging to the upper $J=4$ level is almost pure $K=\frac{9}{2}$ for small $\xi$, but becomes malnly $K=\frac{7}{2}$ for $\xi \cong 2$. Thus In following along the two continuous energy levels with $J=4$, we see 8 
rapid interchange of the composition of the corresponding elgenvectors, iwith a monotonic change in the real "purity". 33 This behavior is a straightforward property of the eigenvalues and vectors of a matrix in which the values of the diagonal elements in a given representation cross as the pertinent parameters are varied. Even when we do not have a case In wich the diagonal elements cross and produce the above type of interchange of elgenvector composition, the diagonal elements may st11l approach equality (as in several cases in F1g. 1), producing very strong imlxing of pure-basis states as the coupling conditions become less appropriate to the representation used. Th1s points up the fact that no Gingle representation can logically be used to designate the nature of quantum states undèr varylng coupling conditions, a situation commonly met within any single spectrum.

One further coment should be ade in regard to these figures. One IImit of Fig. I has been labeled pure LS coupling, and the other only approximately pure LK coupling; the limits of Fig. 2 are pure $j J$ coupling and approximate $j K$ coupling. The reason for this is clear from expressions (3) and (4). In the latter, the $\left(\ell_{2}{ }_{2}\right)$ interaction required to define a unique value of the quantum number $K$ simultaneously destroys the absolute "volidity of the quantum number $L$; and in the former, the $\left(\ell_{1} \ell_{2}\right)$ interaction required to define $K$ deatroys the validity of $J_{1}$. Thus in a sense there is no such thing as pure LK nor pure JK coupling, but only pure pair coupling (5): $K$ well defined by virtue of zero $\left(l_{2} s_{2}\right)$ and $\left(s_{1} b_{2}\right)$ interactions. However, this is somewhat academic since pure LK or $J K$ $+\cdots \div \div \quad \div \quad 21$ 
coupling conditions can in principle be approached arbitrarily closely, and since one in practice never has pure LS nor $j J$ coupling because of the non-zero interactions between the two vectors.

In Figs. 3-5 we show some details of the transition from LK to $\mathrm{JK}$ coupling. We shall discuss only the pure-palr-coupling case in which there is no splitting of the pairs into levels $J=K \pm \frac{1}{2}$, and so need consider only the $\left(\ell_{2} \ell_{2}\right)$ and $\left(\ell_{1}{ }_{2}\right)$ interactions -- 1.e., the parameters $F_{2}$ and $C_{p}$, in terms of which ve define an abscissa

$$
\xi=\zeta_{p} /\left(18 F_{2}+\zeta_{p}\right)
$$

the constant " 18 " belng chosen so as to give equal total splitting in the limits $\xi=0$ and 1. In Fig. 3 we show the six energy levels, each labeled by the appropriate value of $K$, and of course each doubly degenerate with respect to $J$. In the limit $\xi \rightarrow 0$, these six levels coalesce in pairs into the three quadruply-degenerate levels shown at the extreme right of F1g. 1, and we approach pure LK coupling. Note that this limit Is not the same as that at the extreme left of F1g. 1. In the present case the singlets and triplets are not only degenerate but also highly mixed: ${ }^{1,},{ }^{3} \mathrm{D}_{2}$ are mixed in the proportion $40-60$ for the $\left[\frac{3}{2}\right]_{2}$ state and $60-40$ for the $\left[\frac{5}{2}\right]_{2}$ state, and the mixing is even more complete in the cases ${ }^{2,3} \mathrm{~F}_{9}$ and ${ }^{2,3} \mathrm{G}_{4}$ (cP. the LS-LK transformation matrices in Table AI); clearly the opin quantum number has no meaning. Simflarly, in the limit $\xi \rightarrow 1$ we have well defined values $J_{1}=\frac{1}{2}, \frac{3}{2}$ and approach pure $j K$ coupling, 
$i$ but there 18 mixing of $J_{2}=\frac{5}{2}$ and $\frac{7}{2}$; though the $j_{2}$ mixing here 18 much less severe then in the case of $S$ at $\xi=0$, the quantum number $j_{2}$ also has relatively little significance under palr-coupling conditions.

Throughout the intermediate-pair-coupling region $0<\xi<1$, $\mathrm{K}$ remains a good quantum number in all cases. In the present approximation, $L$ and $J_{1}$ also remain good quantum numbers for the two curves $K=\frac{3}{2}$ and $\frac{9}{2}$, as can be seen from the matrices of Table AII and also from the fact 'that the curves in Fig. 3 are stralght lines. However, for the pair of curves with $K=\frac{7}{2}$ the corresponding eigenvectors show strong mixing of the LK basis-states $G\left[\frac{7}{2}\right], F\left[\frac{7}{2}\right]$ and also strong mixing of the $j K$ basisstates ' $\frac{3}{2}\left[\frac{7}{2}\right], \frac{1}{2}\left[\frac{7}{2}\right]$, with a corresponding $108 \mathrm{~s}$ in significance of the quantum numbers $L$ and $J_{1}$. Thls is illustrated in Fig. 4 which shows the composition of the low-energy $K=\frac{T}{2}$ states (either $J$ value); 1. e., the squares of the non-zero components of the elgenvector in the indicated representation. Note that the two states $\left[\frac{7}{2}\right]_{3,4}$ whlch are practically pure $F$ states when $\xi$ is small are much more strongly $G$ than $F$ when $\xi$ is large, and that the states which are pure $G$ for small $\xi$ are mainly $F$ for large $\xi$; 11kewlse, the atates whlch are almost completely $J_{2}=\frac{1}{2}$ for large $\xi$ are mainly $J_{1}=\frac{3}{2}$ when $\xi$ is small, and vice versa. A similar reversal of composition exists for the $\left[\frac{5}{2}\right]$ states, though to a considerably smaller extent -. the "purity"33 (in elther the LK or JK representation) changing from unity to $\frac{4}{9}$ instead of from unity to $\frac{1}{4}$ in going from one coupling extreme to the other (cf. the LK-JK transformation matrices of Table.AI). These are of course just further examples like those discussed 
for F1g. 2, where enersy levels do not cross because of their matual Interaction, but where the quantum-state compositions behave as though the levels did cross.

Simflar reversals and corresponding changes in purity are of course also present when the elgenvectors corresponding to Fig. 3 are expressed in the LS or $3 f$ representations. This is summarized in F1g. 5, where for each of the four representations we show the average purity of the (equally-weighted) ten states $J \neq 1,5$ (the states $J=1$ and 5 being omitted from the average, since they are always 100 percent pure in the one-configuration approximation). The decreases in purity, using the LK and $\mathrm{JK}$ representations, as we depart from $\xi=0$ and 1 , respectively, correspond to mixing of the type shown in F1g. 4. For the IS and $j J$ representations, we have additional singlet-triplet and $j_{2}=\frac{5}{2}, \frac{7}{2}$ mixing such as that discussed in connection with Fig. 3; these result in correspondingly lower purities than for LK and JK.

The point of all this is that the wavefunctions of some states exhibit very large composition changes as $\xi$ is varied, and 1 it is consequently quite inappropriate to use a single coupling notation for all values of $\xi$. In the present case 1 it is true that one can unambiguously distinguish between the two $\left[\frac{7}{2}\right]_{3}$ states (for example) by using elther the $L K$ notation $F\left[\frac{7}{2}\right]_{3}, G\left[\frac{7}{2}\right]_{3}$ or the $J K$ notation $\frac{1}{2}\left[\frac{7}{2}\right]_{3}, \frac{3}{2}\left[\frac{7}{2}\right]_{3}$ if one reads these to mean "the state which in the limit $\xi \rightarrow 0$ becomes an F state," or "In the limit $\xi \rightarrow 1$ becomes a $J_{1}=\frac{1}{2}$ state," etc. However, though either the IK or the JK notation 18 adequate regardless of the value of 
. F for purposes of simply distinguishing between the two states involved, no single notation always denotes the existing type of coupling and the :true nature of the state. In order that the notation have the latter desirable property to the greatest possible degree, it is necessary to use the IK notation only for small $\xi$ and the JK notation only for large $\xi$-. the dividing line in the present case being at about $\xi=0.45\left(\zeta_{p}=\right.$ $\left.15 F_{2}\right)$. 


\section{Machine Calculation of Coupling Type}

In this section we discuss some illustrations of the coupling conditions which may be found in practice, calculated with a second Fortran code RCE2. Th1s code uses the coefficlent matrices from RCEl in the representation of interest, and varies the values of the Slater parameters $F_{k}, G_{k}, \zeta_{1}$ to give the best (least-squares) agreement between the observed energy levels and the computed eigenvalues of the energy matrices. The final elgenvectors are printed out in each of the four representations, giving an indication of the purity of the wavefunctions in each case. The code also calculates the standard deviation of the energy level fit:

$$
\text { STDEV }=\left\{\frac{\sum(\text { error })^{2}}{\text { no. levels - no. parameters }}\right\}^{1 / 2} ;
$$

the ratio of STDEV to the maximum width of the configuration gives a measure of the accuracy of the fit and thereby some 1dea of the probable accuracy of the calculated wavefunctions. Further details of the code w1ll be described elsewhere.

As a f1rst example, we have in plotted in F1g. 3 the energy levels of the $2 p 4 f$, 
$5 f$, $6 f$ configurations of $N$ II. ${ }^{6}$ The pair degeneracy is here removed by small non-zero values of the $G_{k}$, but the splitting 18 so small that conditions are s.t11l very close to pure pair coupling. The values of $\xi$ used in plotting the data vere calculated from (2I) using the least-square parameter values (which are practlcally equal to those given by Eriksson ${ }^{\theta}$ ); however, it is also clear from the experimental energies themselves that the $4 f$ levels lie to the left of the crossing point of the $K=\frac{5}{2}$ and $\frac{9}{2}$ curves, and that the $5 f$ and $6 f$ levels lie to the right of this point. Thus for the $4 f$ configuration LK-coupling notation is preferable (as used by Eriksson in his final paper); however, JK notation would definitely be preferable for the higher configurations (cf. Figs. 4 and 5).

It cannot be emphasized too strongly that when pair-coupling conditions are approximated, as in these N II configurations, LS notation is practically meaningless and therefore quite misleading. For example, the four low levels of the $N$ II $4 f$ configuration have been 11 sted a.s ${ }^{34}$ :

$$
\begin{array}{ll}
{ }^{1} F_{3} & 211030.90 \mathrm{~cm}^{-1} \\
{ }^{3} F_{2} & 211033.71 \\
{ }^{3} F_{3} & 211057.07 \\
{ }^{3} F_{4} & 211061.03
\end{array}
$$

assignments which have been dictated by the orderings 


$$
{ }^{3} F_{2}<{ }^{3} F_{3}<{ }^{3} F_{4}, \quad{ }^{1} F_{3}<{ }^{3} F_{3}
$$

which exist under IS coupling conditions. The "actual" compositions of the $J=3$ states (calculated with the RCE2 code) are given in Table I for both the LS and LK representations. These results show that, if anytining, the low-energy level $\left(K=\frac{5}{2}\right)$ should be the ${ }^{3} F_{3}$ and the high-energy level $\left(K=\frac{7}{2}\right)$ the ${ }^{2} F_{3}$ (cf. also Table AI). However, the plain fact of the matter is that the separation of the two levels is a $\mathrm{K}$ splitting and not an S splitting at all; IS notation really should never be used at all in such a case.

It should be clear from this example that tables which have been prepared $^{35}$ for conversion of (empirical) LS notation in the literature to the appropriate fK notation should be used only for the specifically designated purpose. Only with care can they be applied to similar configurations of other spectra, and they ought never to be used for the reverse purpose of Inferring the LS composition of levels tabulated elsewhere in $\mathrm{jK}$ notation!

As a further example of the care which should be exercised in level classification, we consider the 3plf configuration of P II, in which the levels were primarily labeled in LS notation, but alternative $j K$ designations also given because of a strong tendency toward level pairing. Actually, the coupling 18 closer to LK than to either LS or $3 K$, as shown by the fact that the $\left[\frac{\theta}{2}\right]_{4,5}$ levels lie below the $\left[\frac{5}{2}\right]_{2,3}$ levels, and by 
the calculated average purities $80,88,65$, and 59 percent (and minimum purities 55, 69, 47, and 39 percent) in LS, LK, JK, and JJ coupling, respectively. The fairly large value of $G_{2}\left(G_{2} / F_{2}=0.061\right.$ for P. II $4 f$ as compared with 0.014 for N II $4 f$ ) results in considerable departure from palr coupling (see Fig. 3) and some tendency toward LS coupling, but LK 1s clearly the best. [Note: The large level shifts from the paircoupling positions show in Fig. 3 are due only to departure from pair coupling and not to perturbations, as shown by the fact that the maximum error in the least-square $\mathrm{fit}$ of the enerby levels is $27 \mathrm{~cm}^{-1}(0.02$ on the scale of Fig. 3), which 16 only 1.8 percent of the width of the configuration.] The fact that the coupling is close to LK rather than $\mathrm{JK}$ explains the fact that the observed $(D-G) /(G-F)$ ratio of 0.377 is "surprisingly close to the value 0.350 predicted by Russell-Saunders coupling theory."

Finally, we show in Table II some calculated results for the Ge I spectrum, ${ }^{10}$ Including: the number of levels and parameters (including "Fo") used in the least-square fit, the fit error (22), the ratio of STDEV to the maximum wldts of the configuration, the least-square parameter values,. and the average percent purity of the wavefunctions (average of the squares of the largest eigenvector components ${ }^{38}$ ) In each representation (always omftting elgenvectors for wich there are no others of the same J). We note the following general features of the tabulated results:

(1) The value of $\zeta_{4 p}$ is roughly independent of the con- 
ificuration (as is to be expected from its definition as a. radial integral, 4 since the outer electron has relatively little effect on the 1ntegrand), and approximates the limit: Ing value $1178.24 \mathrm{~cm}^{-2}$ derived from the Ge II spectrum.

(2) For configurations $n_{2} l_{1} n_{2} l_{2}$ with variable $n_{2}$, all parameters $F_{k}, G_{k}, \zeta_{2}$ decrease w1th increasing $n_{2}$, as is to be expected from their definstions. 4 This means that as $n_{2}$ increases, the coupling conditions tend away from LS or IK coupling and toward $j K$ or $j J$ coupling.

(3) For the configurations 4pns 1t follows from (2)-(4) that $I K, j K$, and $j J$ coupling are 1dentical. From the table, it is seen that in Ge I the coupling is closer to these than to LS, even for the lowest of the ps configurations.

(4) All parameters $F_{k}, G_{k}, \delta_{2}$ decrease as $l_{2}$ 1ncreases, 80 that the coupling tends away from LS-LK toward JK-Jj. Moreover, the $G_{k}$ and $S_{2}$ tend to decrease faster than $F_{2}$, so that one tends more and more closely toward the pair-coupling approximation. (The rather large values of $\delta_{2}$ in the pd conPigurations are probably spurious, resulting from perturbations suggested by the rather large errors in the energy fit.)

(5) The average purities are of course approximate, the elgenvectors being sometimes subject to considerable fluctu- 
ations as the result of perturbations from other configurations. However, wh the possible exceptions of the $5 \mathrm{~d}$ and $6 d$ configurations, the accuracy of the energy fits indicates that the eigenvectors are probably not too far off. In the cases of $5 p$ and $4 d$, the coupling is rather hopelessly mixed up. In all other cases the coupling is fairly distinct, and mostly indicates a definite tendency to $j K$ coupling. The old standby LS notation is clearly appropriate only for $4 p^{2}$ and perhaps 4p5s, 4p5p, and 4p4d.

(6) Theoretical values of the parameters $F_{k}, G_{k}, \zeta_{i}$ have been calculated for all these configurations, using Hartree-FockSlater radial wavefunctions obtained with an adaptation of the SHARE program developed by Herman and Skillman. ${ }^{37}$ with the exception of $\zeta_{2}$ for the pd conflgurations, where as already indicated the empirical values are probably perturbed, the theoretical and empirical values show the same general trends, even though the former tend to be ten to fifty percent larger than the latter. Since the theoretical values cannot be expected to have high absolute accuracy (nor, perhaps, can the empirical ones, in the one-configuration approximation), this generally good agreement provides confirmation of the conclusions dram in the preceding paragraph. 
It should be noted that Ge I 18 somewhat unusual in the extent to which $J K$ coupling applies. With increasing atomic number, the $F_{k}$ and $G_{k}$ tend to decrease slowly whereas the $\zeta_{1}$ increase rapidly. ${ }^{38}$ Thus for low atomic number the electrostatic parameters predominate and result in IS coupling except for high values of $n_{2} l_{2}$ (where there is a tendency toward LK or JK coupling); for high atomic number both of the $\zeta_{1}$ are large and result in $f J$ coupling except for $h 1 g h n_{2} l_{2}$ (where the coupling may be $j K$ ). It is only for intermediate $\mathrm{Z}$ that conditions are such, in neutral-atom spectra, as to produce approximate $j K$ coupling in nearly all excited conf1gurations. (For the spectra of 1ons, these conditions probably occur for lower $\mathrm{Z}$ the bigher the stage of lonization. ${ }^{38}$ ) 


\section{Intensities and Selection Rules}

Intensity relations and selection rules have been derived in the literature for JK coupling, ${ }^{39}$ but apparently not for LK coupling. For completeness, we derive pertinent equations for dipole radiation in all four couplings, following Rohrlich 40 but using modern $3 n-j$ notation. W1th wavefunctions of the type (7) for one configuration and similar functions $\left.\mid \beta^{\prime} J^{\prime} M^{\prime}\right)$ for a second conflguration, the transition probability between states of the two configurations is proportional to the square of the dipole moment:

$$
\begin{aligned}
S_{M M^{\prime}}=\sum_{x y z}\left|\left(\beta J M\left|M_{x}\right| \beta^{\prime} J^{\prime} M^{\prime}\right)\right|^{2} & =\sum_{q}\left|\left(\beta J M\left|P_{q}{ }^{(1)}\right| \beta^{\prime} J^{\prime} M^{\prime}\right)\right|^{2}, \\
& =\left|\left(\beta J\|P(1)\| \beta^{\prime} J^{\prime}\right)\right|^{2} \sum_{q}\left|(-1)^{J-M}\left(\begin{array}{ccc}
J & 1 & J^{\prime} \\
-M & q & M^{\prime}
\end{array}\right)\right|^{2} .
\end{aligned}
$$

Here $\vec{M}=-e \sum_{1} \vec{r}_{1}$ 18 the classical dipole vector, $P_{q}^{(1)}$ is the correspond- 
Ing Irreducible tensor (of rank 1 ), 11 and the final form of (23) Involving a reduced matrix element and a 3-j symbol follows from the Wigner-Eckart theorem. 41,25-27 We shall not be concerned here with the MM' dependence of this expression, and consider only the sum of (23) over all the magnetic bub-states, which gives

$$
\stackrel{S}{S}=\sum_{M M^{\prime}} S_{M M^{\prime}}=\left|\left(\beta J\left\|P^{(2)}\right\| \beta^{\prime} J^{\prime}\right)\right|^{2}
$$

since the $M M^{\prime}$ sum of the square of the $3-j$ function $16 \frac{1}{3}$, independently of $\mathrm{J}, \mathrm{J}^{\prime}$, and $\mathrm{q}$.

We consider only transitions of the type $n_{1} l_{2}^{\varepsilon_{2}} n_{2} l_{2}-n_{1} l_{1}^{\epsilon_{2}} n_{2}^{\prime} l_{2}^{\prime}$ in which $n_{2} l_{2} \neq n_{2}^{\prime} l_{2}^{\prime}$. Then only the term $-\vec{e}_{2}$ of $\vec{M}$ contributes to (24), and for this $\stackrel{S}{ }$ is diagonal in the quantum numbers $\alpha_{1} S_{1} L_{1}$ characterizing the states of the subconfiguration $\ell_{1}^{\epsilon}$. For expansions of the type (7), the reduced dipole-matrix element in (23) or (24) can be expanded in terms of similar matrix elements for pure-coupling wavefunctions:

$$
D_{\beta} \equiv\left(\beta J\left\|P^{(1)}\right\| \beta^{\prime} J^{\prime}\right)=\sum_{a b} \sum_{a}\left(B_{a b J}^{\prime}\right)^{*}\left(a b J\|P(1)\| a^{\prime} b^{\prime} J^{\prime}\right) B_{a^{\prime} b^{\prime} J^{\prime}},
$$

where $a b=L S, L K, J_{2} K$, or $J_{1} J_{2}$. The eigenvector components $B_{a b J}^{\beta}$ are given, for example, by the energy-level-fit calculation described in Sec. VI. The pure-coupling matrix elements for the four representations are easily 
evaluated with the a1d of Racah's equations, ${ }^{42}$ and written in terms 'of $6-j$ symbols are

$$
\begin{aligned}
& D_{M S} \equiv\left(\alpha_{2} L_{1} S_{1}, l_{2} L S, J\|P(1)\| \alpha_{1} L_{1} S_{2}, l_{2} L^{\prime} S^{\prime}, J^{\prime}\right)
\end{aligned}
$$

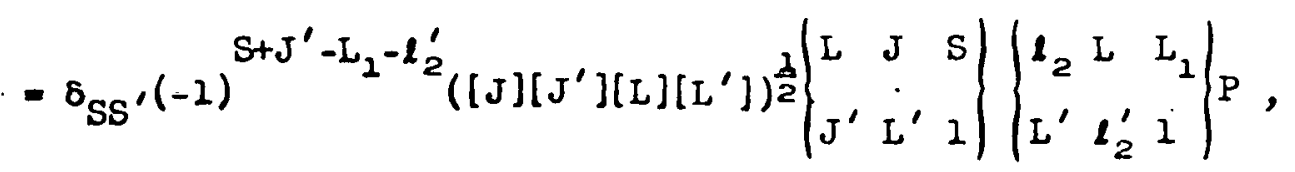

$$
\begin{aligned}
& D_{L K} \equiv\left(\left(\alpha_{1} I_{1} l_{2}\right) L, S_{2} K, s_{2} J\|P(1)\|\left(\alpha_{1} L_{2} l_{2}^{\prime}\right) L^{\prime}, S_{1} K^{\prime}, 8_{2} J^{\prime}\right) \\
&=(-1)^{K+8_{2}+J^{\prime}+S_{1}+K^{\prime}-L_{1}-l_{2}^{\prime}-1}\left([J]\left[J^{\prime}\right][K]\left[K^{\prime}\right][L]\left[L^{\prime}\right]\right)^{\frac{1}{2}} \\
&\left|\begin{array}{lll}
K & J & s_{2} \\
J^{\prime} & K^{\prime} & 1
\end{array}\right|\left|\begin{array}{lll}
L & K & S_{1} \\
K^{\prime} & L^{\prime} & 1
\end{array}\right|\left|\begin{array}{lll}
l_{2} & L & L_{1} \\
L^{\prime} & l_{2}^{\prime} & 1
\end{array}\right| P,
\end{aligned}
$$

$$
\begin{aligned}
& {\stackrel{D}{J_{1} K}}^{D_{1}} \equiv\left(\alpha_{1} L_{1} S_{2} J_{1}, l_{2} K, 8_{2} J\|P(1)\| \alpha_{1} L_{1} S_{1} J_{1}^{\prime}, l_{2}^{\prime} K^{\prime}, 8_{2} J^{\prime}\right)
\end{aligned}
$$

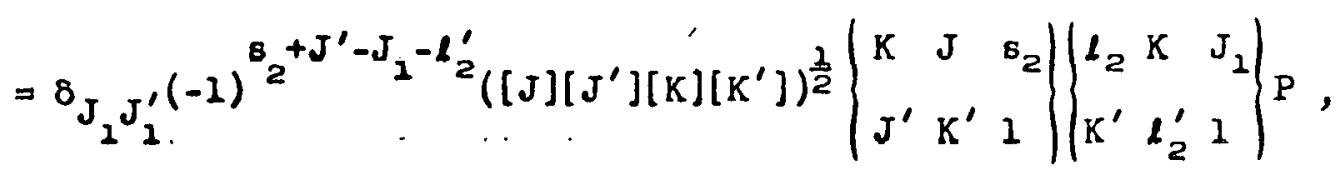




$$
\begin{aligned}
D_{J_{2} J_{2}} & \equiv\left(\alpha_{1} I_{1} S_{2} J_{1}, \ell_{2} B_{2} J_{2}, J\|P(1)\| \alpha_{1} I_{1} S_{2} J_{1}^{\prime}, l_{2}^{\prime} s_{2} J_{2}^{\prime}, J^{\prime}\right) \\
& =\delta_{J_{1} J_{1}^{\prime}}(-1)^{J_{1}+J-\ell_{2}-8_{2}}\left([J]\left[J^{\prime}\right]\left[J_{2}\right]\left[J_{2}^{\prime}\right]\right)^{\frac{1}{2}}\left|\begin{array}{lll}
J_{2} & J & J_{1} \\
J^{\prime} & J_{2}^{\prime} & 1
\end{array}\right|\left\{\begin{array}{lll}
l_{2} & J_{2} & s_{2} \\
J_{2}^{\prime} & L_{2}^{\prime} & 1
\end{array}\right) P .
\end{aligned}
$$

In these expressions $P$ is the reduced matrix element ${ }^{43}$

$$
\begin{aligned}
& P \equiv\left(n_{2} l_{2}\left\|-e \vec{r}_{2}\right\| n_{2}^{\prime} l_{2}^{\prime}\right) \\
& =(-1)^{l_{2}}\left(\left[l_{2}\right]\left[\ell_{2}^{\prime}\right]\right)^{\frac{1}{2}}\left(\begin{array}{lll}
l_{2} & 1 & l_{2}^{\prime} \\
0 & 0 & 0
\end{array}\right) \int_{0}^{\infty}(-e r) R_{l_{2}}(r) R_{l_{2}^{\prime}}(r) d r \\
& =\delta_{l_{2}^{\prime}, l_{2}+1}(-1)^{l_{2}+l_{>}} l_{\gamma^{2}} \int_{0}^{\frac{1}{2}}(-e r) R_{l_{2}}(r) R_{l_{2}^{\prime}}(r) d r ;
\end{aligned}
$$


the general selection rule

$$
l_{2}^{\prime}=l_{2} \pm 1
$$

arises from properties of the 3-j symbol in (30). Other selection rules arise very simply from a property of $6-\mathrm{J}$ symbols such that

$$
\left(\begin{array}{lll}
a & b & c \\
d & e & f
\end{array} \mid=0\right.
$$

unless each of the triads $(a b c),(a e f),(d b f)$, and (dec) satisfies the triangle relation (i.e., no element of the triad is greater than the sum of the other two). In this way we find the selection rule

$$
\Delta J=0, \pm 1 \quad\left(J=J^{\prime}=0 \text { not allowed }\right)
$$

holding in all cases (26) to (29), and find the following selection rules holding whenever the quantum number in question is relevant to the purecoupling case involved:

$$
\begin{aligned}
\Delta L=0, \pm 1 & \left(\mathrm{~L}=\mathrm{L}^{\prime}=0 \text { not allowed }\right) \\
\Delta \mathrm{K}=0, \pm 1 & \left(\mathrm{~K}=\mathrm{K}^{\prime}=0 \text { not allowed }\right) \\
\Delta J_{2}=0, \pm 1 & \left(J_{2}=J_{2}^{\prime}=0 \text { not allowed }\right)
\end{aligned}
$$


of course, the additional selection rules $\Delta S=0$ and $\Delta J_{1}=0$ follow from the delta functions in the pertinent equations. Most of these selection rules are well known, but we have felt it worthwhile to point out how simply they follow from the Racah treatment and the triangle-rule property of the 6-j symbol. In instances where the coupling is not close to one of the four pure cases, the selection rules of course become invalid to greater or lesser extent because of the mixing of pure-basis states implicit in (25).

Under pure-coupling conditions it follows from Eqs. (24) and (25) that Ine strengths are proportional to the square of the appropriate expression (26)-(29). Varlous sum rules, some well known and some not, then follow very simply from a normalization property of 6-j symbols ${ }^{27}$; for example,

$$
\sum_{J^{\prime}}[J]\left[J^{\prime}\right]\left\{\begin{array}{lll}
X & J & Y \\
J^{\prime} & X^{\prime} & 1
\end{array}\right\}^{2}=\frac{[J]}{[X]}
$$

from which also

$$
\sum_{J} \sum_{J^{\prime}}[J]\left[J^{\prime}\right]\left\{\begin{array}{lll}
X & J & Y \\
J^{\prime} & X^{\prime} & 1
\end{array}\right\}^{2}=[X]^{-2} \sum_{J}[J]=[Y] .
$$

Repeated use of expressions analogous to (36) shows that if $\mathrm{S}$ is summed over $J, J^{\prime}$ and all intermediate quantum numbers, then we find for the total 
Ine strength of all transitions between the two configurations

$$
\sum_{N}=\left[s_{1}\right]\left[\tau_{1}\right]\left[s_{2}\right]\left|\left(l_{2}\|P(1)\| \ell_{2}^{\prime}\right)\right|^{2}
$$

which, as 1t should be, is independent of the coupling.

As an example of relative line strengths in pair coupling, we show In Tables III and IV values for the transition pf $-p g\left(\right.$ or $p^{5} f-p^{5} g$ ) in both pure LK and pure JK coupling. (The level scheme for pg is qualitatively identical with Fig. 3 except that all values of $L$ and $K$ are greater by unity.) For simplicity, we have considered only sums (of the squares of (27) and (28)] over $J$ and $J^{\prime}$, which is consistent with the absence of $J$ splitting in pure pair coupling, and have multiplied by a convenience factor of $2^{2} 3^{4} 7^{2} / P^{2}$ to give integral results. Certain sums over various intermediate quantum numbers are shown in parentheses in various blocks of the tables and opposite the rows and columns of the tables; they are exanples of varlous sum relations derivable similarly to (35).

Note that the intensity patterns in these tables are such that there are very few strong line pairs available for finding constant wavenumber differences, thus making it difficult to locate levels of such configurations under pair coupling conditions. Moreover, the patterns in the two limiting couplings are not sufficiently different to provide a definite basis for decision on the type of pair coupling existing in a given case, on the basis of Intensity considerations alone. This is more easily done 
on the basis of the relative positions of the energy levels (cf. the levels $K=\frac{9}{2}$ and $K=\frac{5}{2}$ in Fig. 3 ). 


\section{Weak-f1eld Zeeman Effect}

A further test of the accuracy of wavefunctions such as those calculated in connection with Table II can be made by using them to compute values of the Lande g-factor.

If the atom Iles in a magnetic field of "intensity $\mathcal{H}$, then there must be added to (14) a magnetic energy

$$
H_{\text {mag }}=\frac{e f C}{2 m c} \sum_{1}\left(L_{z_{1}}+2 s_{z_{1}}\right)=\frac{e F C}{2 m c}\left(J_{z}+s_{z}\right) .
$$

In this fleld only $M$ is a constant of the motion and, in principle, complete wavefunctions are obtained only as linear comblnations of the functions ( 7 ) for flxed $M$ but all $J \geqq M$; however, in the limit of very weak flelds the added terms are negligible and we can use the functions (7) without change. It is easily seen that the magnetic energy for the state $k=$ BJM is given in units of $e N / 2 m c$ by the matrix product

$$
g^{k}=\sum_{1 j}\left(B_{1}^{k}\right)^{*}\left(B_{1 j}\right)\left(B_{j}^{k}\right)
$$


where $\mathrm{g}^{\mathrm{k}}$ (not to be confused with the quantity defined in (15)1) is the g-factor of the state $k$, and the $B_{1}^{k}$ are the elgenvector components (coefficients in the expansion (7)), for any specific representation. As is well known, the g-matrix

$$
B_{1 j} \equiv\left(1 / J_{2}+S_{2}(j) / M\right.
$$

is easily evaluated in the IS representation, where it is completely diagonal with diagonal elements

$$
B_{11} \equiv B_{L S J}=1+\frac{J(J+1)-L(L+1)+S(S+1)}{2 J(J+1)} .
$$

Values of the $g$-factors for each state $k$ can readily be found from (39) by using (41) and, if necessary, transforming the eigenvectors into the IS representation by means of the appropriate matrix from Sec. III. However, it is instructive to instead transform the matrix (41) into the representation of interest by means of the transformation $\mathrm{U}^{*} \mathrm{gU}$. (Tnis is the procedure actually followed in the RCE code.) Then if the coupling is pure, we see from (39) that the $g^{k}$ are just the corresponding diagonal elements $g_{k k}$ of the G-matrix; if the coupling is not pure, the degree of departure from purity is indicated by the degree to which the $g^{k}$ depart from the $\mathrm{g}_{\mathrm{kk}^{\circ}}{ }^{44}$ Unfortunately, there exist almost no experimental data on 
g-factors in pe configurations; consequently, we use $p p^{\prime}$ (or rather $p^{5} p^{\prime}$ ) as an example: In Table $V$ are shown the complete $g$-matrices for $J=I$ in the four representations. In Table VI we list the diagonal elements $8_{\mathrm{kk}}$ for all levels $J \neq 0$ in each representation. The correlation of states In the different representations has been made according to the dominant elements of the three transformation matrices $L S \rightarrow L K, L K \rightarrow j K, j K \rightarrow j j$; this correlation results in close correlation of the values $g_{k k}$ between representations. If the correlation of states had been made on the basis of the continulty of energy levels (in going from LK to JK coupling) similarly to Fig. 3 for $\mathrm{pf}$, then all $P$ and $D$ states would have been correlated with $J_{2}=\frac{1}{2}$ and $\frac{3}{2}$, respectively; this would have reversed the correlations for ${ }^{3} D$ and ${ }^{1} P\left(K=\frac{3}{2}, J=1\right)$ and for ${ }^{3} D$ and ${ }^{3} P\left(K=\frac{3}{2}, J=2\right)$ for reasons entirely analogous to Fig. 4, and would have spoiled the correlation between the $g_{k k}$ for LS or LK coupling and those for $j K$ or $S j$ coupling. Also shown in Table VI are experimental values of the $g^{k}$ for the two lowest $p^{5} p^{\prime}$ configurations of the rare gases, ${ }^{45}$ together with correspondIng values calculated from (39) using wavefunctions derived as described in Section VI from least-square fits of the energy levels. We note the following:

(1) Calculated g-factors agree reasonably well with the observed values.

(2) Both the $\bar{g}$-factors and the calculated average purities Indicate strong departures from LS coupling in all cases, and 
a definite tendency toward $J K$ coupling -- especially for the higher configuration (smaller $F_{k}, G_{k}$, and $\sigma_{p}$ ) and for higher $z$ (lorger $\left.\delta_{p}\right)$.

(3) In the case of $\mathrm{Ne} I \mathrm{3p}$, where the coupling may be expected to be closest to IS or LK and farthest from $j K-j J$, the observed $\&$ values clearly indicate incorrect assignments for the four $K=\frac{3}{2}$ levels. This is because the $g$ values were entered in Table VI according to the $j K$ assignments listed in $\mathrm{AEL}^{34}$; if the more appropriate IK notation had been used, then the level with $8=0.999$ would have been designated $P\left[\frac{3}{2}\right]$ rather than $\frac{1}{2}\left[\frac{3}{2}\right]$, etc., and all $g$ values would have come out in the proper order. Here we see direct experimental confirmation of composition reversals like those shown in Fig. 4,46 and see again the danger in exclusive use of one type of coupling notation to designate the nature of a state, even though this may be adequate to 1dentify an energy level (cf. the remarks at the end of sec. V). 
FOOTNOTES

* Work performed partially under the auspices of the U. S. Atomic Energy Commision and partially under a grant of the National Science Foundat1on.

1 1'G. Racah, J. Opt. Soc. Am. 50, 408 (1960).

2 A. G. Shenstone, Phil. Trans. Roy. Soc. London A235, 195 (1936).

3 G. H. Shortley and B. Fried, Phys. Rev. 24,749 (1938).

4. U. Condon and G. H. Shortley, The Theory of Atomic Spectra (Cambridge Univ. Press, 1935), especially Chaps. 6-7, 10-11, 13.

5 J. C. Slater, Quantum Theory of Atomic Structure (McGraw-H111 Book Co., Inc., New York, 1960), especially Chaps. 12-13, 24. For brief discussions of the theory, see also H. G. Kuhn, Atomic Spectra (Academic Press, New York, N.Y., 1962), Chap. V, and reference 22, Sec. II.

k. B. S. Eriksson; Phys. Rev. 102, 102 (1956); Arkiv Fysik 13, 303 (1958). 7 I. J. Radzlemsk1, Ph. D. Thes1s, Purdue Univ., 1964. 
${ }^{B}$ Y. G. Toresson, Arkiv Fysik 18, 389 (1960).

8

W. C. Mart1n, J: Opt. Soc. Am 49, 1071 (1959).

10 K. L. Andrew and K. W. Melssner, J. Opt. Soc. Am. 49, 146 (1959); C. J. Humphreys and K. L. Andrew, J. Opt. Soc. Am. 54, Aug. (1964).

11

A. M. Crooker, private communication.

12 W. G. Brill, Ph.D. Thesis, Purdue Univ., 1964; J. Opt. Soc. Am. 54,566 (1964).

13

B. Edlén and J. W. Swensson, reported at Atomic Spectroscopy Symposium, Argonne National Laboratory, June, 1961.

14

N. Spector, J. Opt. Soc. Am. 53, 1349 (1963).

15

K. B. S. Eriksson and I. Johansson, Arkiv Fysik 19, 235 (1961).

16

K. B. S. Eriksson, Arkiv Fysik 19, 229 (1961).

27

L. Minnhagen, Arkiv Fysik 18, 97 (1960).

28

I. Minnhagen, J. Opt. Soc. Am. 21, 298 (1961).

29

G. Racah, Phys. Rev. 61, 537 (1942).

20

Reference 4, p. 123.

21

N. H. Möller, Arkıv Fys1k 18, 135 (1960). 


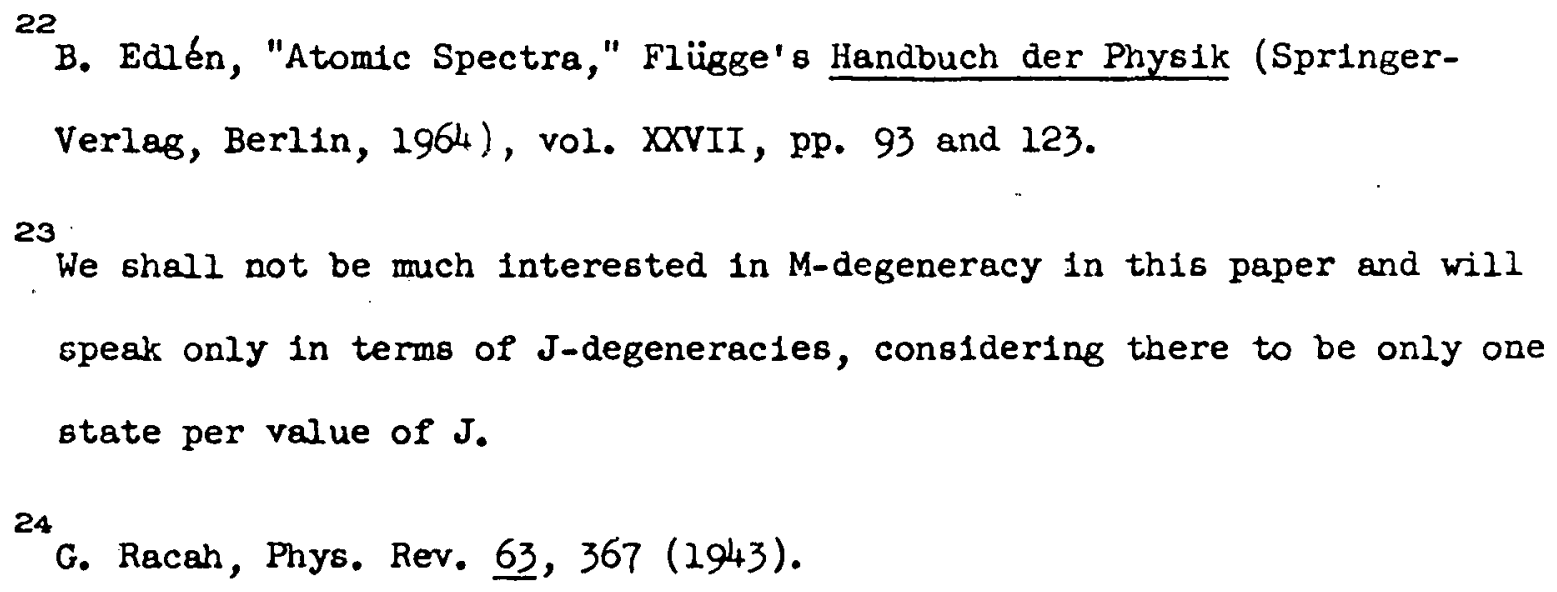


AII.

33

G. H. Shortley, Phys. Rev. 44,670 (1933).

34

C. E. Moore, Atomic Energy Levels (NBS Circular 467, U. S. Govt. Printing Off., Washington, D. C. , vol. I, 1949), p. 36.

35

For example, reference 34, vol. II (1952), p. 116.

36

Note that this 18 different from the definition of purity used in discussing Figs. 4 and 5, and accounts for the increase in apparent LS and IK purity in going from the $4 f$ to the $6 f$ configuration.

37

F. Herman and S. Skillman, Atomic Structure Calculations (Prentice-Hall, Inc., Englewood Cliffs, New Jersey, 1963).

38

Reference 22, Tables 38 and 42 and F1g. 12.

39

L. Minnhagen, Arkiv Fysik 1, 425 (1949).

40

F. Rohrlich, Astrophys. J. 129, 441,449 (1959).

42

G. Racah, Phys. Rev. 62,438 (1942).

42

Reference 41, Eqs. (44); or reference 25, Eqs. (7.1.7) and (7.1.8).

43

E. g., reference 26, Eq. (4-4).

44

The diagonal elements in any representation can be calculated directly by means described by J. C. van den Bosch, Flügge's Handbuch der Physik 
(Springer-Verlag, Berlin, 1957), vol. XXVIII, pp. 305f, but the offdiagonal elements are readily found only by transforming the 8 -matrix from the IS representation.

45 Data from AEI, reference 34; those for XeI have been omitted because they are badly perturbed: J. B. Green, E. H. Hurlburt, and D. W. Bowman, Phys. Rev. 59, 72 (1941).

48

J. B. Green, Phys. Rev. 52, 736 (1937). 
Table I. Calculated Composition of the Low-Energy $J=3$ States of N II 2p4f. (Values in parentheses are for the paircoupling approximation, $\left.G_{2}=G_{4}=\zeta_{f}=0.\right)$

\begin{tabular}{|c|c|c|c|c|}
\hline Level $\left(\mathrm{cm}^{-1}\right)$ & ${ }^{1_{F_{3}}}$ & ${ }^{3} \mathrm{~F}_{3}$ & ${ }^{3} \mathrm{D}_{3}$ & ${ }^{3} \mathrm{G}_{3}$ \\
\hline 211030.90 & $\left\{\begin{array}{l}0.45 \\
(0.40)\end{array}\right.$ & $\begin{array}{c}0.49 \\
(0.54)\end{array}$ & $\begin{array}{c}0.06 \\
(0.06)\end{array}$ & $\begin{array}{r}0.00 \\
(0)\end{array}$ \\
\hline 211057.07 & $\left\{\begin{array}{l}0.45 \\
(0.50)\end{array}\right.$ & $\begin{array}{c}0.42 \\
(0.38)\end{array}$ & $\begin{array}{r}0.00 \\
(0)\end{array}$ & $\begin{array}{c}0.13 \\
(0.12)\end{array}$ \\
\hline 221030.90 & $\frac{F\left[\frac{7}{2}\right]_{3}}{0.00}$ & $\frac{F\left[\frac{5}{2}\right]_{3}}{0.94}$ & $\frac{D\left[\frac{5}{2}\right]_{3}}{0.06}$ & $\frac{G\left[\frac{7}{2}\right]_{3}}{0.00}$ \\
\hline 211057.07 & 0.87 & 0.00 & 0.00 & 0.13 \\
\hline
\end{tabular}


Table II: Least Square Paraneter Values and Coupling Type in Ge I

(STDEV and parameter values in $\mathrm{cm}^{-1}$ )

\begin{tabular}{|c|c|c|c|c|c|c|c|c|c|c|c|c|c|}
\hline & No. & No. & & STDEV & $F$ & & G. & $C$ & & Ave. & Purity & (per & ent) \\
\hline Conf. & Lev. & Par. & STDEV & WIDIH & ${ }^{5} 2$ & $v_{2-1}$ & $v_{l+1}$ & $s_{4 p}$ & ${ }^{\delta} n l$ & $\underline{\underline{\mathrm{LS}}}$ & $L K$ & $j K$ & $j j$ \\
\hline $4 p 5 s$ & 4 & 3 & 1.2 & $0.05 \%$ & - & - & 576 & 1110 & -- & 87 & 94 & 94 & 94 \\
\hline 4068 & 4 & 3 & 86.8 & 4.3 & -- & -- & 158 & 1213 & -- & 74 & 99 & 99 & 99 \\
\hline $4 p 7 s$ & 4 & 3 & 44.7 & 2.3 & - & -- & 116 & 1203 & - & 72 & 100 & 100 & 100 \\
\hline $4 \mathrm{p} 8 \mathrm{~s}$ & 4 & 3 & 7.6 & 0.41 & -- & - & 62 & 1178 & - & 70 & 100 & 100 & 100 \\
\hline 4pgs & 4 & 3 & 42.7 & 2.4 & -- & -- & 51 & 1149 & -- & 69 & 100 & 100 & 100 \\
\hline $4 p^{2}$ & 5 & 3 & 40.6 & 0.25 & 1016 & -- & -- & 906 & -- & 99 & -- & -- & 65 \\
\hline $4 p 5 p$ & 10 & 6 & 21.3 & 0.42 & 148 & 915 & 14 & 1144 & 105 & 73 & 72 & 77 & 73 \\
\hline $4 p 5 p$ & 20 & 6 & 14.6 & 0.60 & 48 & 303 & 5 & 1155 & 34 & 65 & 71 & 86 & 87 \\
\hline $4 p 7 p$ & 10 & 6 & 9.3 & 0.41 & 22 & 160 & 6 & 1173 & 10 & 63 & 68 & 86 & 91 \\
\hline $4 p 4 d$ & 12 & 6 & 135.0 & 3.1 & 196 & 172 & 7.6 & 1190 & 40.6 & 71 & 63 & 73 & 7,4 \\
\hline $4 p 5 d$ & 12 & 6 & 214.3 & 7.9 & 92 & 58 & 3.6 & 1129 & 69.6 & 64 & 68 & 85 & 84 \\
\hline $4 p 6 d$ & 12 & 6 & 95.4 & 4.1 & 19 & 35 & 1.0 & 1206 & 57.6 & 56 & 59 & 83 & 92 \\
\hline $4 p 4 f$ & 12 & 6 & 3.1 & 0.16 & 8.5 & 0.2 & 0.0 & 1176 & -0.4 & 50 & 66 & 95 & 92 \\
\hline $4 p 5 f$ & 12 & 6 & 2.4 & 0.13 & 4.1 & 0.1 & -0.1 & 1175 & -0.0 & 52 & 70 & 99 & 94 \\
\hline $4 p 61$ & 9 & 6 & 0.2 & 0.01 & 2.2 & 0.1 & -0.0 & 1180 & -0.2 & 52 & 71 & 99 & 95 \\
\hline
\end{tabular}


Table III. Relative Line Strengths for $p f-p g$ (or $p^{5} f-p^{5} g$ ) Transitions in LK Coupling

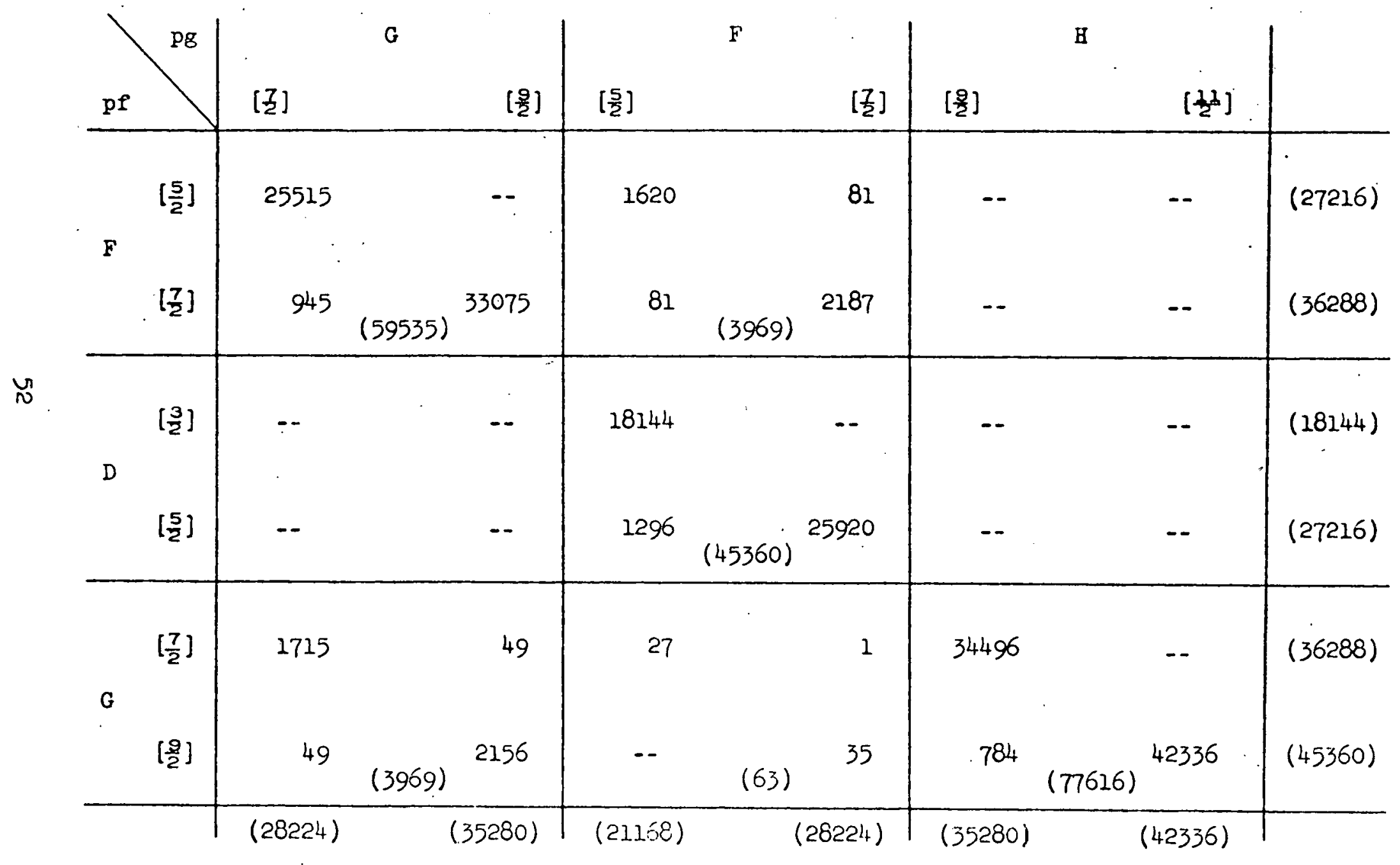


Table IV. Relative Line Strengths for pf - pg (or $p^{5} f-p^{5} g$ ) Transitions in jK Coupling

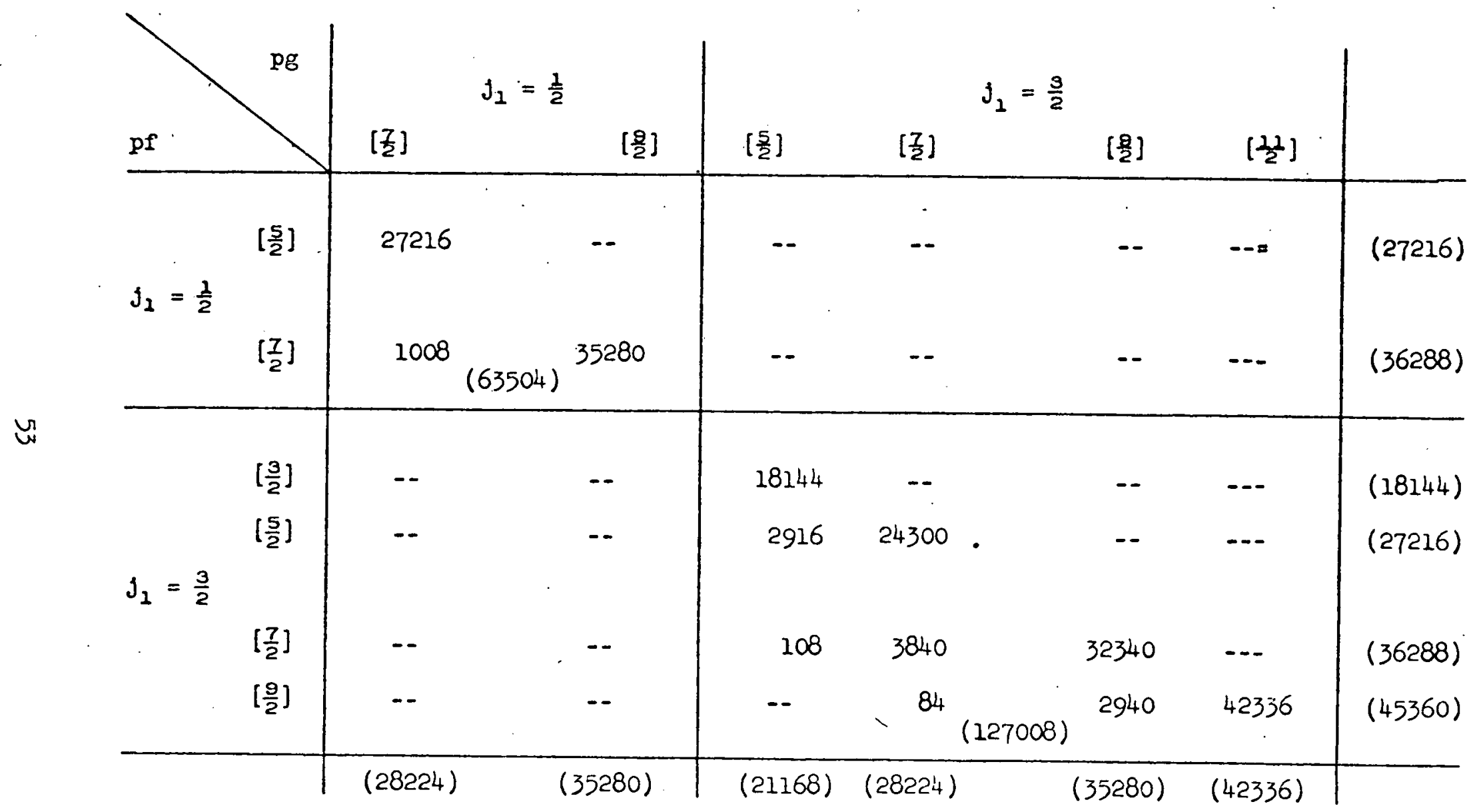


Table V. $J=1$-Matrices for $p p^{\prime}$ or $p^{5} p^{\prime}$ in the Representations LS, $L K, j K, j J$
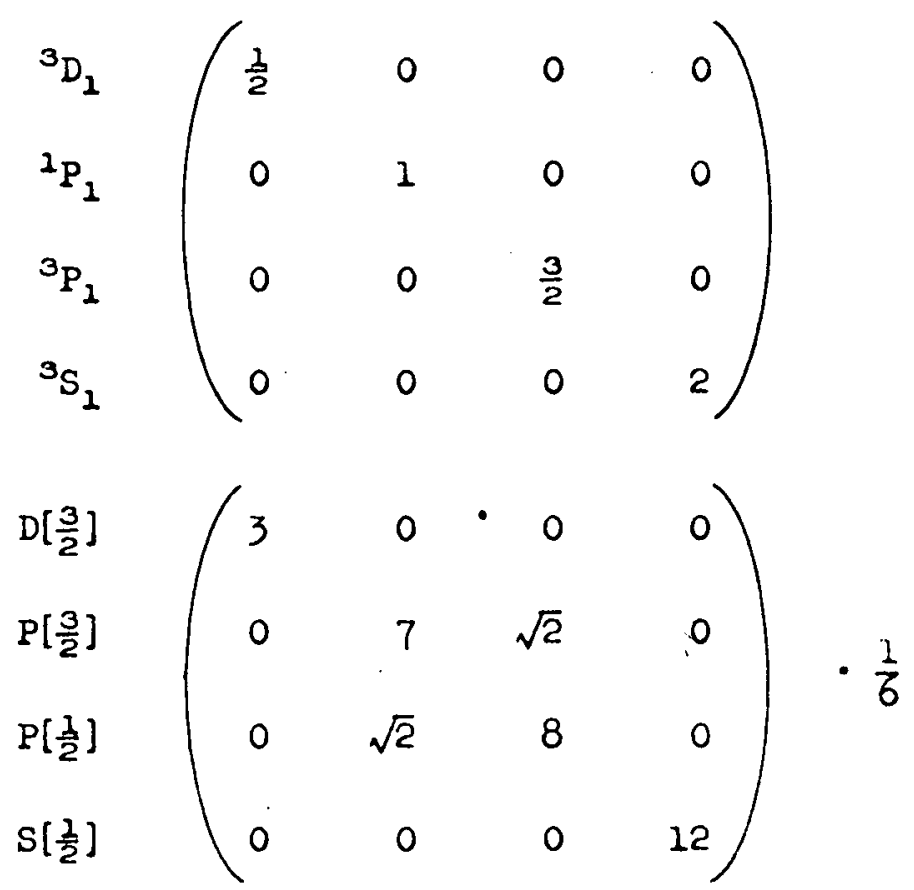

$\frac{1}{2}\left[\frac{3}{2}\right]$
$\frac{3}{2}\left[\frac{3}{2}\right]$
$\frac{1}{2}\left[\frac{1}{2}\right]$
$\frac{3}{2}\left[\frac{1}{2}\right]$$\left(\begin{array}{cccc}11 & 2 \sqrt{5} & \sqrt{2} & -1 \\ 2 \sqrt{5} & 19 & \sqrt{10} & -\sqrt{5} \\ \sqrt{2} & \sqrt{10} & 28 & 4 \sqrt{2} \\ -1 & -\sqrt{5} & 4 \sqrt{2} & 32\end{array}\right) \cdot \frac{1}{18}$

$\left(\frac{1}{2} \frac{1}{2}\right)$
$\left(\frac{3}{2} \frac{3}{2}\right)$
$\left(\frac{1}{2} \frac{3}{2}\right)$
$\left(\frac{3}{2} \frac{1}{2}\right)$$\left(\begin{array}{cccc}4 & 0 & -\sqrt{2} & \sqrt{2} \\ 0 & 8 & \sqrt{5} & -\sqrt{5} \\ \sqrt{2} & \sqrt{5} & 9 & 0 \\ \sqrt{2} & \sqrt{5} & 0 & 9\end{array}\right) \cdot \frac{1}{6}$


Table VI. Landé B-Factors, Least-Sçuare Pararizters, and Coupling Type tn $\mathrm{p}^{5} \mathrm{p}^{\prime}$

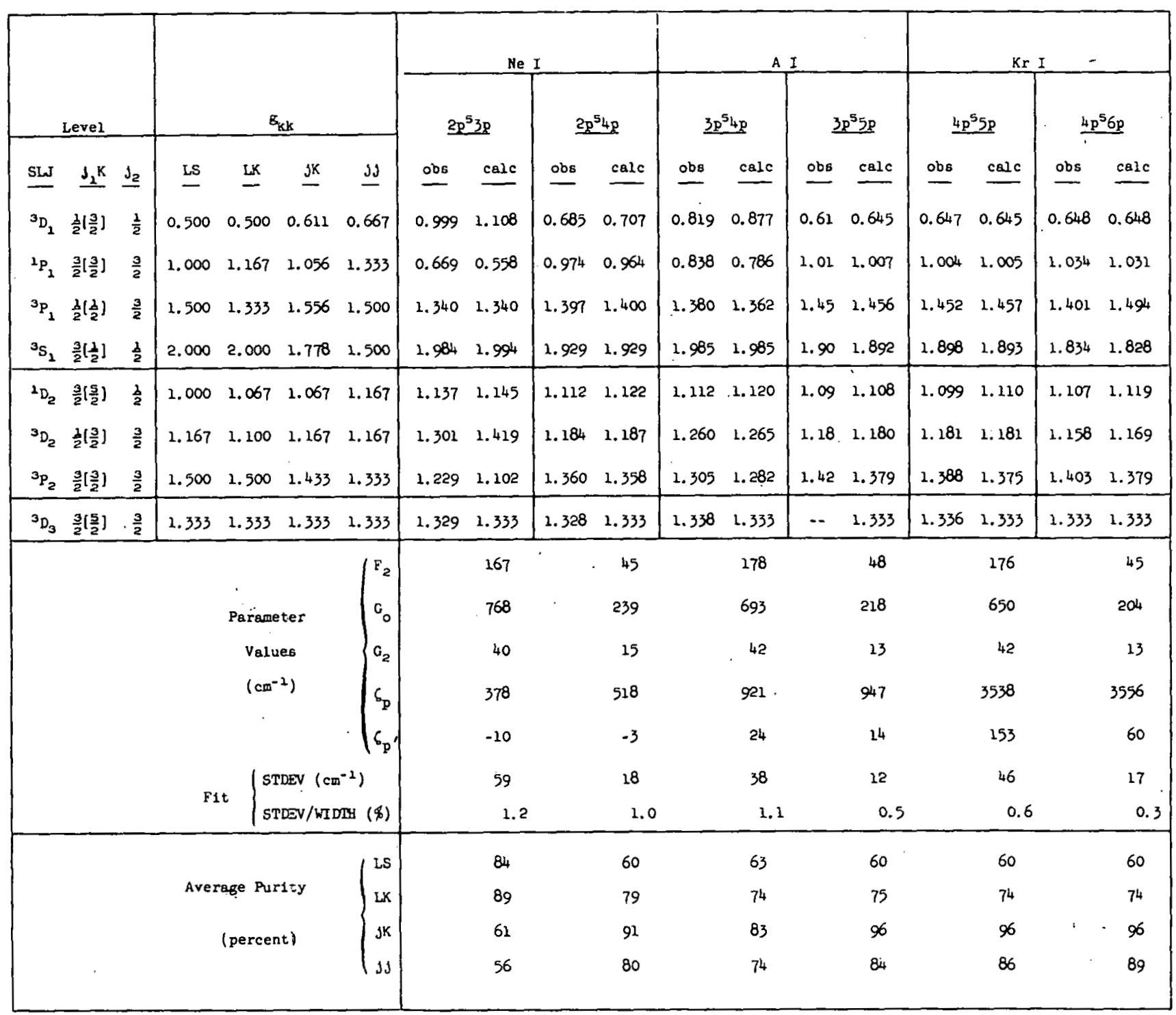


Table AI. Transformation Matrices for $p f, p^{5} f$, or $f^{13} p$. (Matrices for the LS - JK, LK - JJ, and LS - JJ transformations are readily obtalnable by multiplication of those given here.)

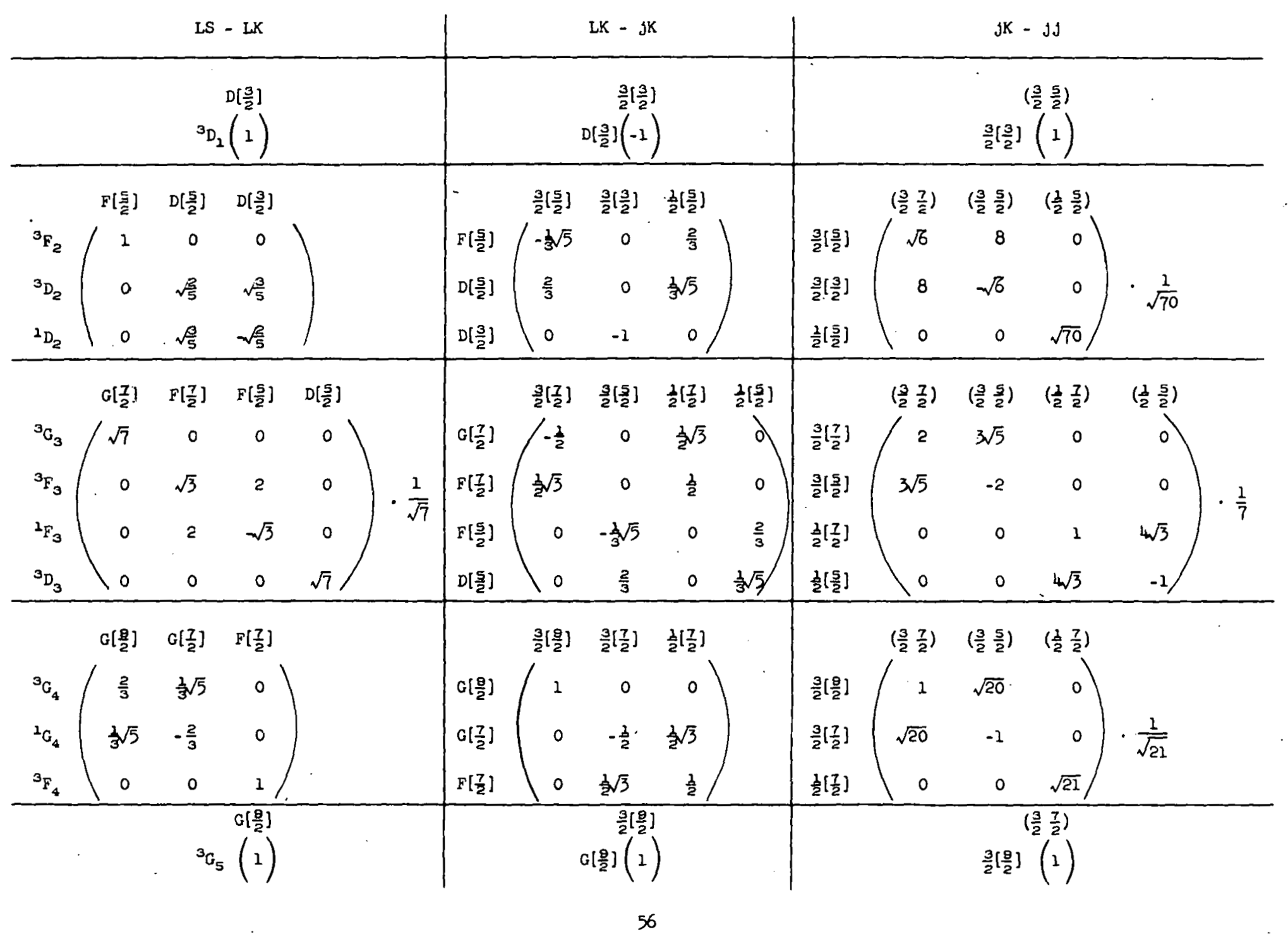


Table AII. Coefficient Matrices for pf (Coefficients are for $F_{2}, G_{2}, G_{4}, \zeta_{p}, \zeta_{f}$, in that order)

LS Coupling

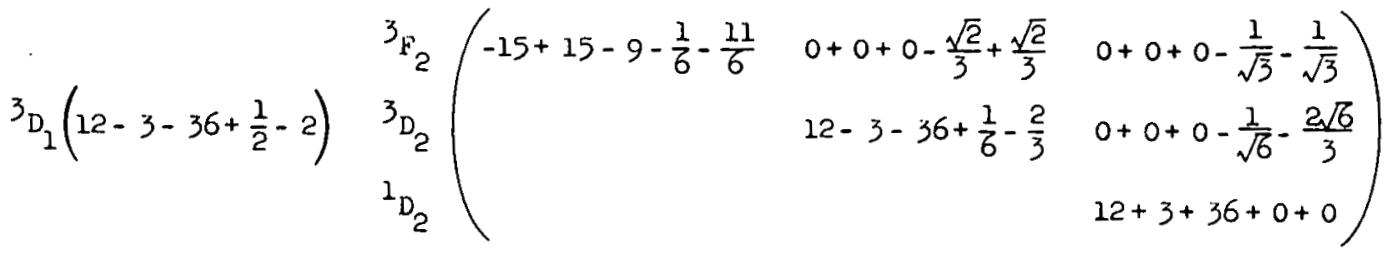

$$
\begin{aligned}
& \begin{array}{l}
3_{G_{3}} \\
3_{F_{3}} \\
I_{F_{3}} \\
3_{D_{3}}
\end{array}\left(\begin{array}{cccc}
5-45-1-\frac{5}{8}-\frac{15}{8} & 0+0+0-\frac{9}{8 \sqrt{7}}+\frac{9}{8 \sqrt{7}} & 0+0+0-\frac{3}{4} \sqrt{\frac{3}{7}}-\frac{3}{4} \sqrt{\frac{3}{7}} & 0+0+0+0+0 \\
& -15+15-9-\frac{1}{24}-\frac{11}{24} & 0+0+0+\frac{1}{4 \sqrt{3}}-\frac{11}{4 \sqrt{3}} & 0+0+0-\sqrt{\frac{20}{63}}+\sqrt{\frac{20}{63}} \\
& -15-15+9+0+0 & 0+0+0+\sqrt{\frac{5}{21}}+\sqrt{\frac{5}{21}}
\end{array}\right)
\end{aligned}
$$

$$
3_{F_{4}}^{{ }_{G_{4}}}{ }_{G_{4}}\left(\begin{array}{ccc}
5-45-1-\frac{1}{8}-\frac{3}{8} & 0+0+0+\frac{\sqrt{5}}{4}+\frac{3 \sqrt{5}}{4} & 0+0+0-\frac{\sqrt{15}}{8}+\frac{\sqrt{15}}{8} \\
& 5+45+1+0+0 & 0+0+0+\frac{\sqrt{3}}{4}+\frac{\sqrt{3}}{4} \\
& -15+15-9+\frac{1}{8}+\frac{11}{8}
\end{array}\right){ }^{3} G_{5}\left(5-45-1+\frac{1}{2}+\frac{3}{2}\right)
$$

$\underline{\text { LK Coupling }}$

$D\left[\frac{3}{2}\right]\left(12-3-36+\frac{1}{2}-2\right)$

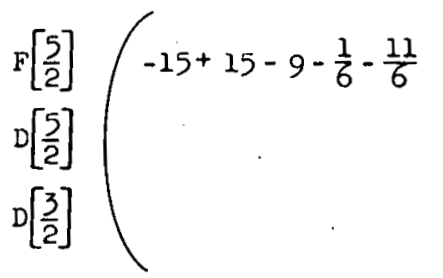

$G\left[\frac{7}{2}\right] / 5-45-1-\frac{5}{8}-\frac{15}{8}$

$F\left[\frac{7}{2}\right]$

$F\left[\frac{5}{2}\right]$

$D\left[\frac{5}{2}\right]$

$$
0+0+0-\frac{3 \sqrt{3}}{8} \ldots \frac{3 \sqrt{3}}{56}
$$

$-15-\frac{15}{7}+\frac{9}{7}+\frac{1}{8}-\frac{99}{56}$

$$
\begin{aligned}
& 0+0+0+0+\frac{9}{14} \\
& 0+\frac{60 \sqrt{3}}{7}-\frac{36 \sqrt{3}}{7}+0-\frac{11 \sqrt{3}}{42} \\
& -15+\frac{15}{7}-\frac{9}{7}-\frac{1}{6}+\frac{55}{42}
\end{aligned}
$$

$12+\frac{3}{5}+\frac{36}{5}-\frac{1}{3}-\frac{28}{15}$

$$
\left.\begin{array}{l}
0+0+0+0+2 \sqrt{\frac{2}{15}} \\
0-\frac{6 \sqrt{6}}{5}-\frac{72 \sqrt{6}}{5}+0-\frac{4 \sqrt{6}}{15} \\
12-\frac{3}{5}-\frac{36}{5}+\frac{1}{2}+\frac{6}{5}
\end{array}\right)
$$

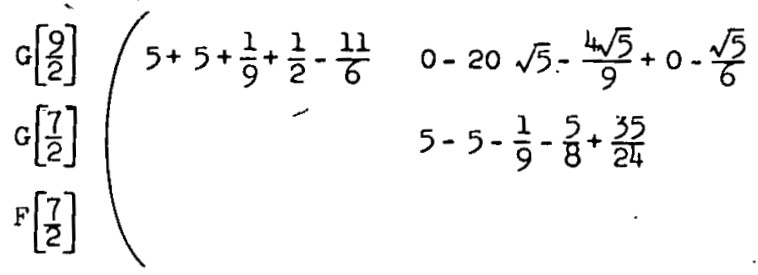

$$
\left.\begin{array}{l}
0+0+0+0+\frac{\sqrt{15}}{6} \\
0+0+0-\frac{3 \sqrt{3}}{8}+\frac{\sqrt{3}}{24} \\
-1.5+15-9+\frac{1}{8}+\frac{11}{8}
\end{array}\right)
$$

$0+0+0+0+0$

$0+0+0+0+\frac{4 \sqrt{15}}{21}$

$0+0+0-\frac{\sqrt{5}}{3}+\frac{\sqrt{5}}{21}$ $12-3-36-\frac{1}{3}+\frac{4}{3}$

$G\left[\frac{9}{2}\right]\left(5-45-1+\frac{1}{2}+\frac{3}{2}\right)$ 
Table AII (continued).

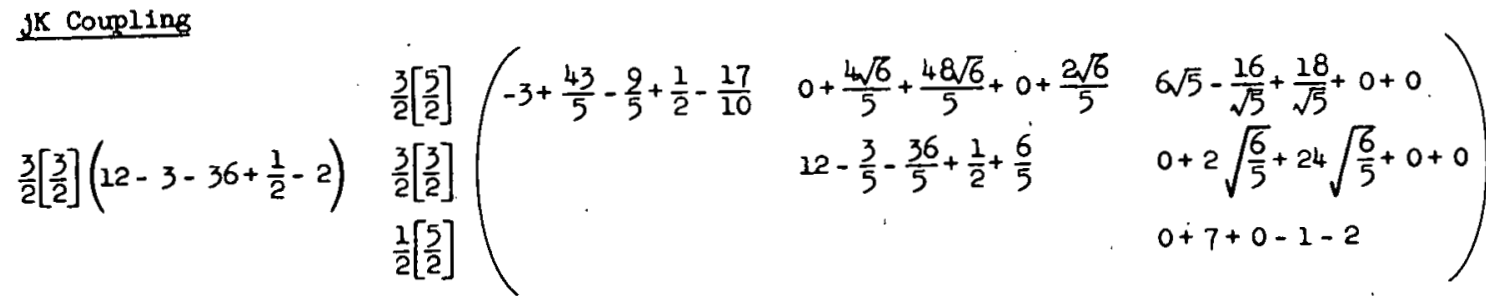

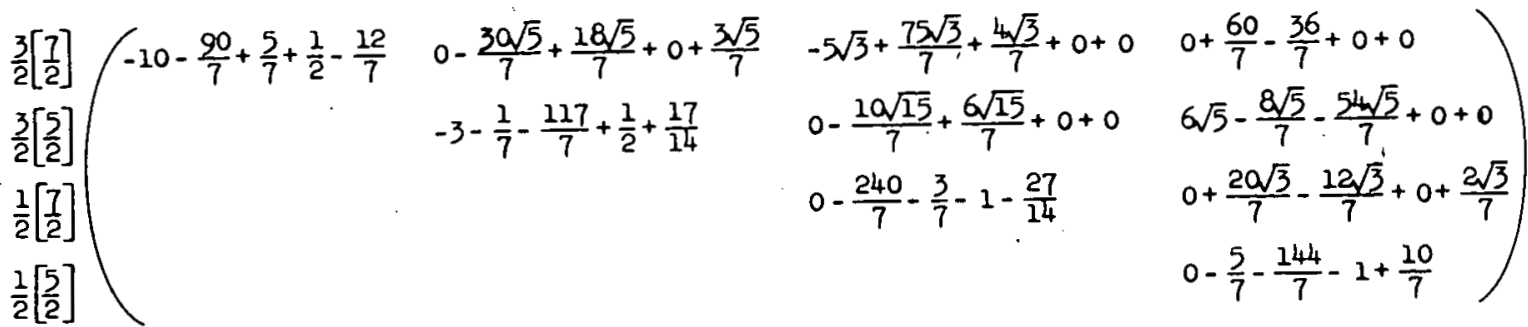

$\frac{3}{2}\left[\frac{9}{2}\right]$
$\frac{3}{2}\left[\frac{7}{2}\right]$
$\frac{1}{2}\left[\frac{7}{2}\right]$$\left(\begin{array}{ccc}5+5+\frac{1}{9}+\frac{1}{2}-\frac{11}{6} & 0+10 \sqrt{5}+\frac{2 \sqrt{5}}{9}+0+\frac{\sqrt{5}}{3} & 0-10 \sqrt{15}-\frac{2 \sqrt{15}}{9}+0+0 \\ & -10+10-\frac{61}{9}+\frac{1}{2}+\frac{4}{3} & -5 \sqrt{3}+5 \sqrt{3}-\frac{20 \sqrt{3}}{9}+0+0 \\ & 0+0-\frac{7}{3}-1+\frac{3}{2}\end{array}\right) \frac{3}{2}\left[\frac{9}{2}\right]\left(5-45-1+\frac{1}{2}+\frac{3}{2}\right)$

jj. Coupling

$\left.\begin{array}{ll}\left(\frac{3}{2} \frac{5}{2}\right)\left(12-3-36+\frac{1}{2}-2\right) & \left(\frac{3}{2} \frac{5}{2}\right) \\ & \left(\frac{1}{2} \frac{5}{2}\right)\end{array}\right)\left(\begin{array}{ccc}7+\frac{12}{7}+\frac{47}{7}-\frac{108}{7}+\frac{1}{2}-2 & 24 \sqrt{\frac{2}{7}}-\sqrt{56}+0+0+0 \\ & 0+7+0-1-2\end{array}\right)$

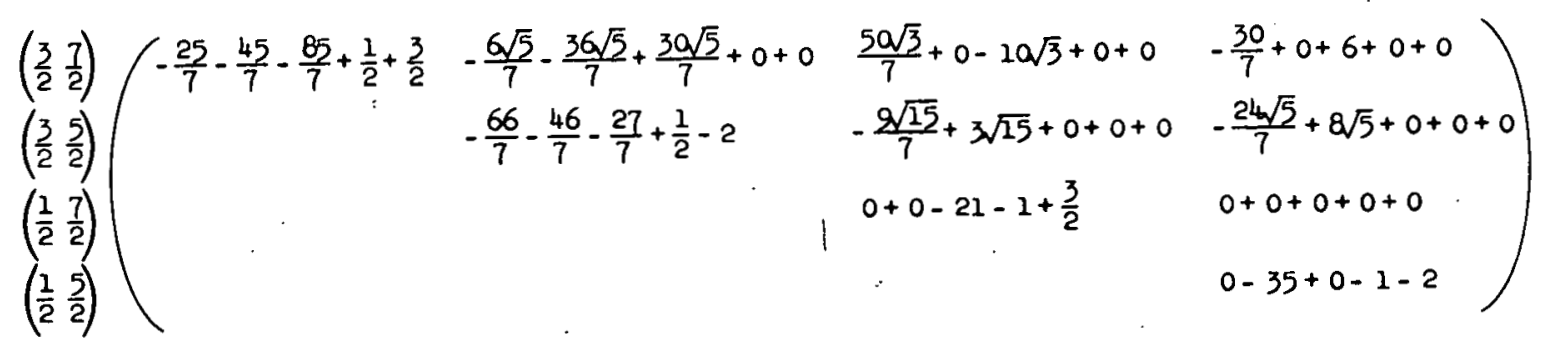

$\left(\begin{array}{ccc}\frac{3}{2} \frac{7}{2}\end{array}\right)\left(\begin{array}{ccc}-\frac{65}{7}+\frac{135}{7}-\frac{131}{21}+\frac{1}{2}+\frac{3}{2} & \frac{10 \sqrt{5}}{7}+\frac{60 \sqrt{5}}{7}+\frac{6 \sqrt{5}}{7}+0+0 & -10 \sqrt{\frac{5}{7}}+0-\frac{2 \sqrt{35}}{3}+0+0 \\ 30 & 30-3 & 1\end{array}\right)$

$\frac{30}{7}-\frac{30}{7}-\frac{3}{7}+\frac{1}{2}-2$

$\frac{5}{\sqrt{7}}-15 \sqrt{7}+0+0+0$

$\left(\frac{3}{2} \frac{7}{2}\right)\left(5-45-1+\frac{1}{2}+\frac{3}{2}\right)$

$\left(\frac{1}{2} \frac{7}{2}\right)$ 
Table AIII. Coefficient Matrices for $p^{5} f$ Coefficients for $F_{2}, \zeta_{p}$, and $\zeta_{f}$ are respectively $-1,-1$, and +1 times those for pf. The only nonzero coefficients for $G_{2}$ and $G_{4}$ are as shown below.

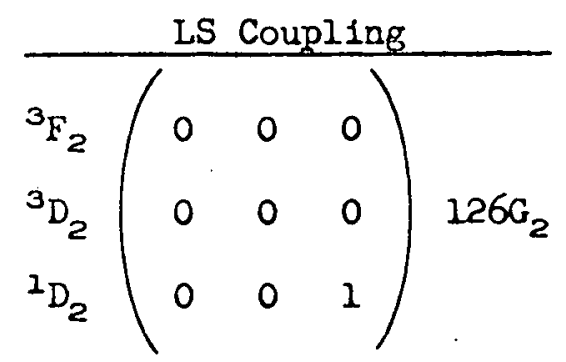
JK Coupling

$\frac{3}{2}\left[\frac{5}{2}\right]$
$\frac{3}{2}\left[\frac{3}{2}\right]$
$\frac{1}{2}\left[\frac{5}{2}\right]$$\left(\begin{array}{ccc}4 & 2 \sqrt{6} & 2 \sqrt{5} \\ 2 \sqrt{6} & 6 & \sqrt{30} \\ 2 \sqrt{5} & \sqrt{30} & 5\end{array}\right) \frac{42 G_{2}}{5}$

${ }_{G_{4}}^{{ }^{1} G_{4}}\left(\begin{array}{ccc}0 & 0 & 0 \\ 0 & 1 & 0 \\ 0 & 0 & 0\end{array}\right) 56 G_{4}$

$\frac{3}{2}\left[\frac{9}{2}\right]$
$\frac{3}{2}\left[\frac{7}{2}\right]$
$\frac{1}{2}\left[\frac{7}{2}\right]$$\left(\begin{array}{ccc}5 & \sqrt{5} & -\sqrt{15} \\ \sqrt{5} & 1 & -\sqrt{3} \\ -\sqrt{15} & -\sqrt{3} & 3\end{array}\right) \frac{56 G_{4}}{9}$

LK Coupling

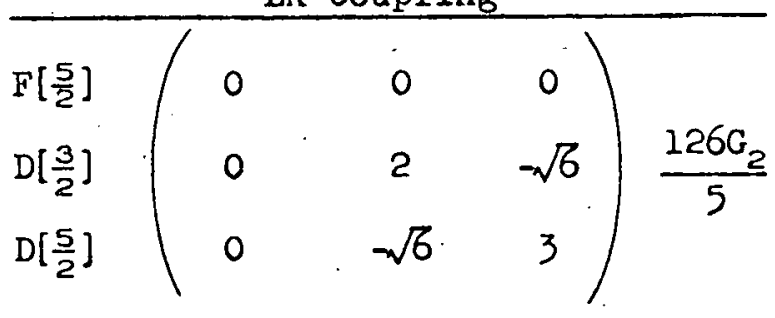

$G\left[\frac{9}{2}\right]$
$G\left[\frac{7}{2}\right]$
$F\left[\frac{7}{2}\right]$$\left(\begin{array}{ccc}5 & -2 \sqrt{5} & 0 \\ -2 \sqrt{5} & 4 & 0 \\ 0 & 0 & 0\end{array}\right) \frac{56 G_{4}}{9}$ j.) Couplings

$\begin{array}{ccc}\left(\frac{3}{2} \frac{7}{2}\right) & \left(\frac{3}{2} \frac{5}{2}\right) \\ \left(\frac{1}{2} \frac{5}{2}\right)\end{array}\left(\begin{array}{ccc}12 & 2 \sqrt{6} & 2 \sqrt{21} \\ 2 \sqrt{6} & 2 & \sqrt{14} \\ 2 \sqrt{21} & \sqrt{14} & 7\end{array}\right) 6 G_{2}$

$\left(\frac{3}{2} \frac{7}{2}\right)$
$\left(\frac{3}{2} \frac{5}{2}\right)$
$\left(\frac{1}{2} \frac{7}{2}\right)$$\left(\begin{array}{ccc}5 & 3 \sqrt{5} & -\sqrt{35} \\ 3 \sqrt{5} & 9 & -3 \sqrt{7} \\ -\sqrt{35} & -3 \sqrt{7} & 7\end{array}\right) \frac{8 G_{4}}{3}$ 


\section{Figure Captions}

Fig. 1. The development of the level structure of the configuration pf for both LS-coupling $(\xi \cong 0)$ and LK coupling $(\xi \cong 1)$ conditions, and the transition from one extreme to the other for the special case $F_{2}=1$, $50 G_{2}+S_{p}=3, G_{4}=\delta_{f}=0$. Note the inverted ${ }^{3} \mathrm{D}$, and that as $\xi$ increases the triplet level which splits off to pair with the singlet is the level $\dot{J}=\mathrm{L}+1$ for ${ }^{3} \mathrm{G}$ and the level $\mathrm{J}=\mathrm{L}-1$ for ${ }^{3} \mathrm{D}$ and ${ }^{3} \mathrm{~F}$.

Fig. 2. The development of the level structure of the conflguration pf for $j K$-coupling $(\xi \cong 0)$ and $j j-c o u p l i n g(\xi \cong 1)$ conditions, and the transition between the two extremes for the special case $\zeta_{p}=1,5 \mathrm{~F}_{2}+$ $\zeta_{P}=0.15, G_{2}=G_{4}=0$. Note that the two highest $J=4$ levels, and the two lowest $J=3$ levels, would cross if it were not for the interaction between them.

Fig. 3. The energy-level structure of the configuration pf in pair coupling $\left(G_{k}=S_{f}=0\right)$. The twelve J-states of this configuration correspond to six doubly-degenerate levels, each characterized by a value of $[K]$ and with $J=K \pm \frac{1}{2}$. In the limit $\xi \rightarrow 0$, pure LK-coupling. exists and the three quadruply-degenerate, levels correspond to $L=F, G$, 
$D$; in the limtt $\xi \rightarrow 1$, the coupling is pure $j_{1} K$, with one quadruplydegenerate $J_{1}=\frac{1}{2}$ level and one eight-fold degenerate $j_{1}=\frac{3}{2}$ level. As examples, the positions of the levels of P II $3 p 4 f$ and of N II $2 p 4 f-6 f$ are plotted at the appropriate $\xi$ values.

F1g. 4. The composition of the lower-energy $K=\frac{7}{2}$ states (either $J=3$ or 4 , and any $M$ value) of the configuration pf. The solid curves give the composition when using the LK representation (e.g., at $\xi=0.2$, these states are each 96 percent $F\left[\frac{7}{2}\right]$ and 4 percent $\left.G\left[\frac{7}{2}\right]\right)$. Similarly, the dashed curves give the composition when using $J K$ basis functions (e. g. , at $\xi=0.2$ the states are 40 percent $\frac{1}{2}\left[\frac{7}{2}\right]$ and 60 percent $\frac{3}{2}\left[\frac{7}{2}\right]$ ). (The compositions of the higher-energy $K=\frac{7}{2}$ states are the complements of the low-energy compositions; 1.e., they are given by interchanging the $F$ and $G$ labels, and interchanging the $j_{1}$ values, in the figure.)

118. 5. The average purity of the elgenvectors of the pf configuration (averaged over the ten levels $J \neq 1,5$ ), for each of the four representations LS, LK, JK, $3 j$. 


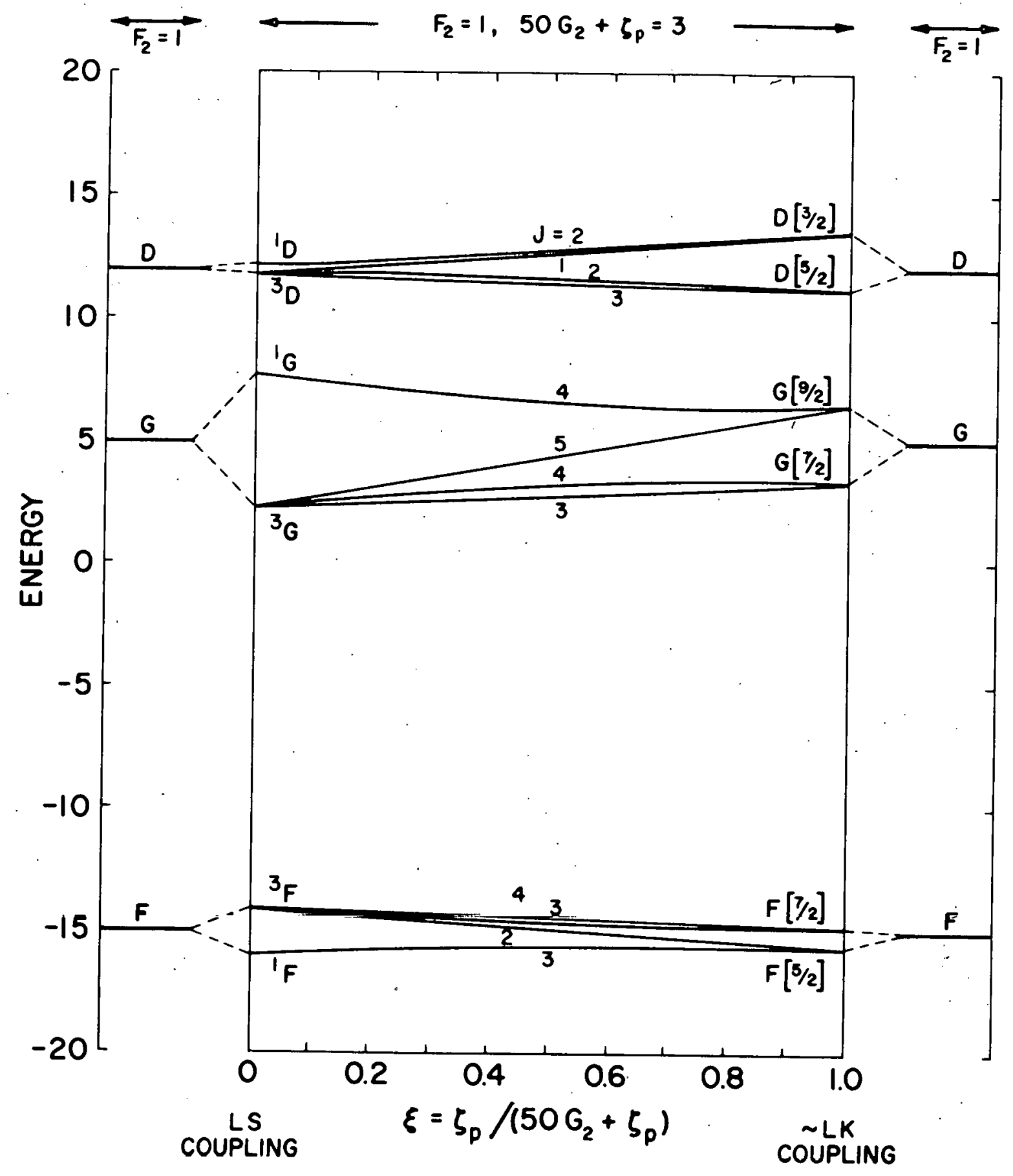

FIG. I 


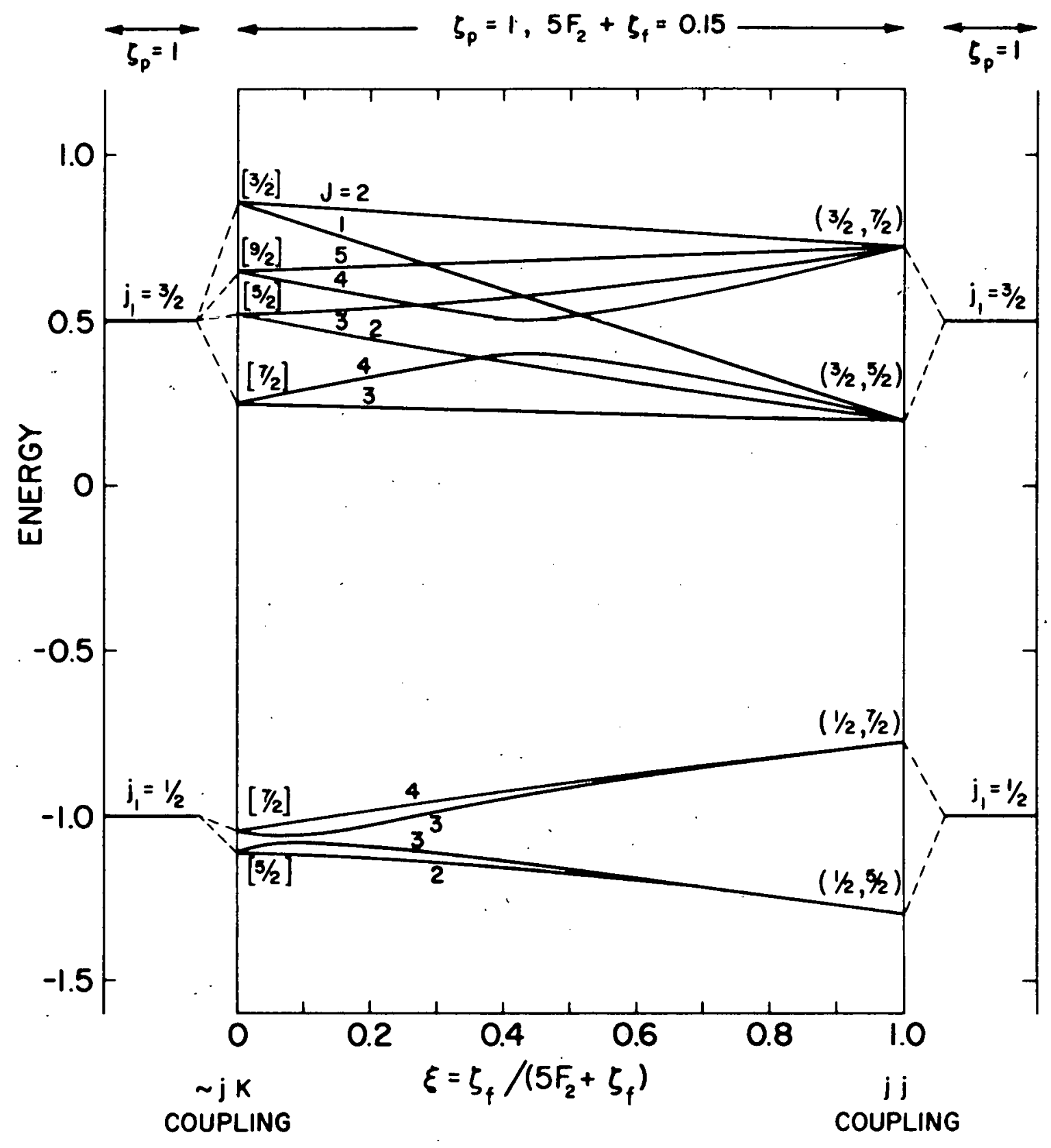

FIG. 2 


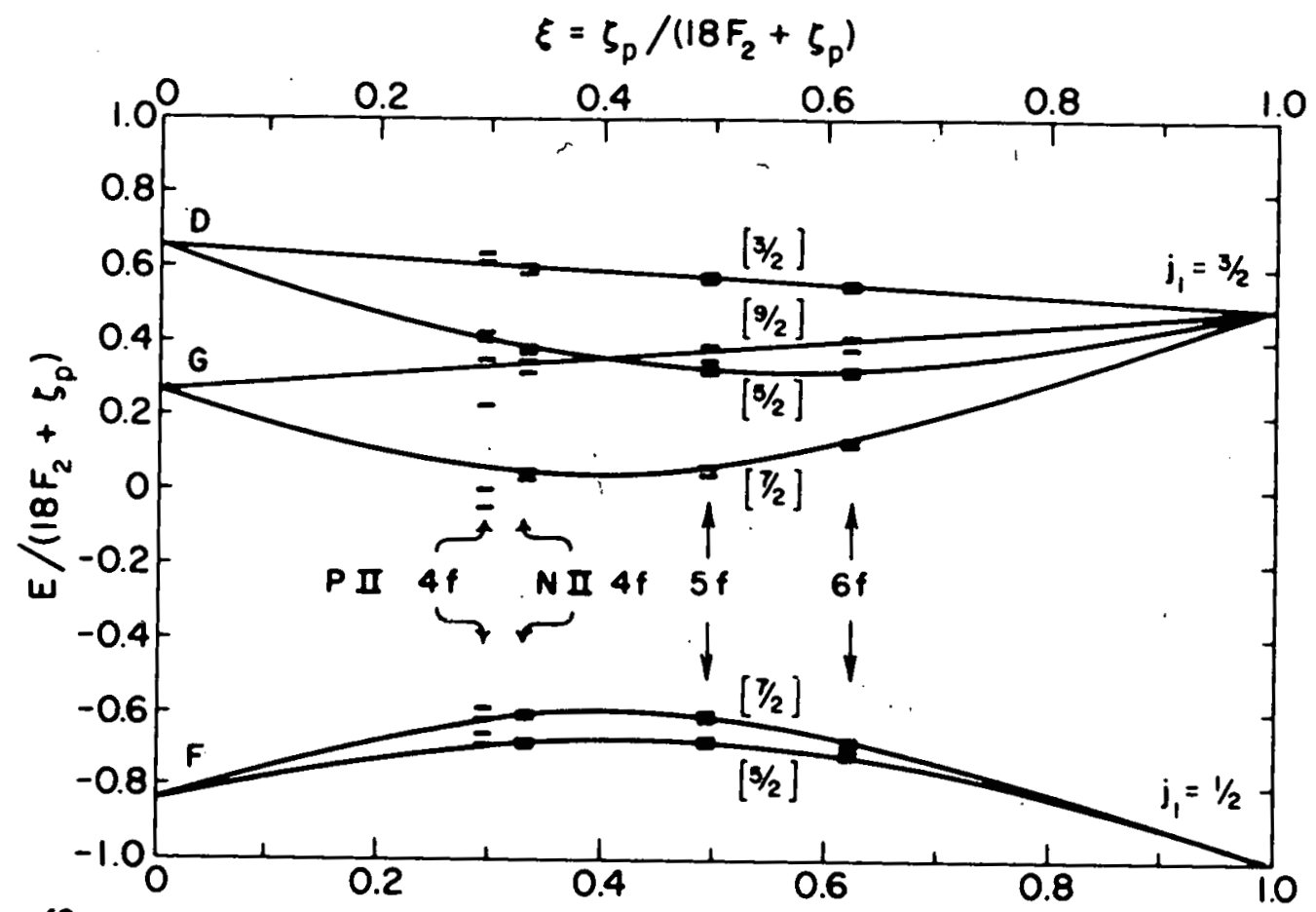

FIG. 3

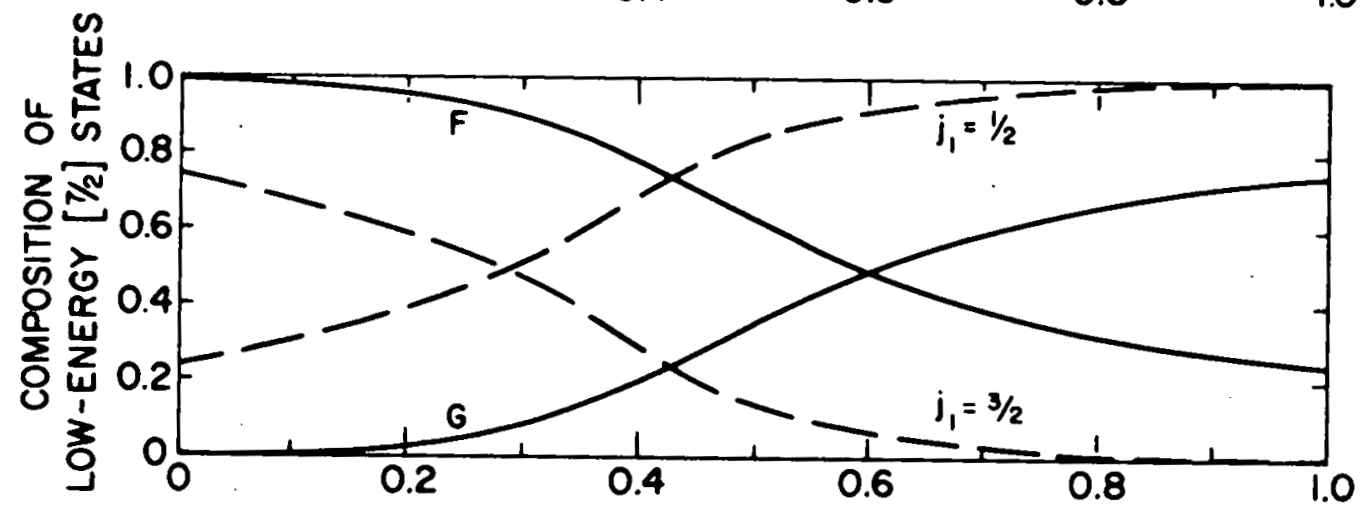

FIG. 4

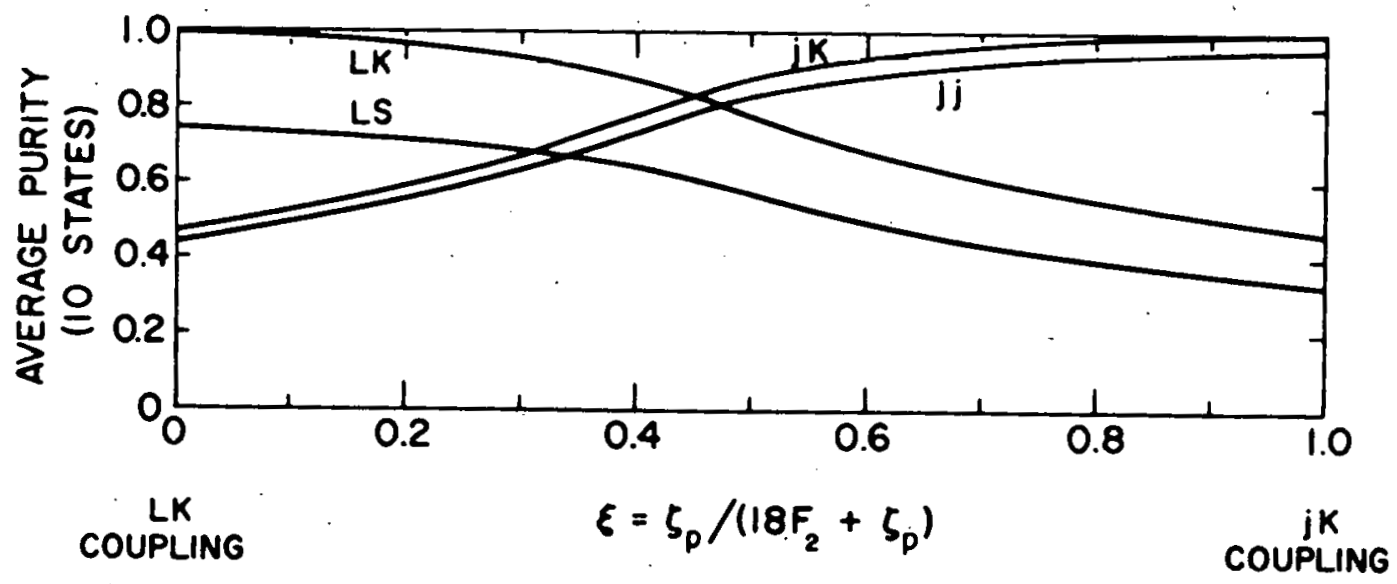

FIG. 5 